\title{
GENERALIZED GAUDIN MODELS AND RICCATIANS
}

\author{
ALEKSANDER GEORGE USHVERIDZE \\ Department of Theoretical Physics, University of Eódź \\ Pomorska 149/153, PL-90-236 Eódź, Poland \\ E-mail: alexush@mvii.uni.lodz.pl and alexush@krysia.uni.lodz.pl
}

\begin{abstract}
The systems of differential equations whose solutions exactly coincide with Bethe ansatz solutions for generalized Gaudin models are constructed. These equations are called the generalized spectral $\left({ }^{1}\right)$ Riccati equations, because the simplest equation of this class has a standard Riccatian form. The general form of these equations is $R_{n_{i}}\left[z_{1}(\lambda), \ldots, z_{r}(\lambda)\right]=c_{n_{i}}(\lambda), i=$ $1, \ldots, r$, where $R_{n_{i}}$ denote some homogeneous polynomials of degrees $n_{i}$ constructed from functional variables $z_{i}(\lambda)$ and their derivatives. It is assumed that $\operatorname{deg} \partial^{k} z_{i}(\lambda)=k+1$. The problem is to find all functions $z_{i}(\lambda)$ and $c_{n_{i}}(\lambda)$ satisfying the above equations under $2 r$ additional constraints $P z_{i}(\lambda)=F_{i}(\lambda)$ and $(1-P) c_{n_{i}}(\lambda)=0$, where $P$ is a projector from the space of all rational functions onto the space of rational functions having their singularities at a priori given points. It turns out that this problem has solutions only for very special polynomials $R_{n_{i}}$. Simplest polynomials of such sort are called Riccatians. One of most important results of the paper is the observation that there exist one-to-one correspondence between the systems of Riccatians and simple Lie algebras. In particular, the degrees of Riccatians associated with a given simple Lie algebra $\mathcal{L}_{r}$ of rank $r$ coincide with the orders of corresponding Casimir invariants. In the paper we present an explicit form of Riccatians associated with algebras $A_{1}, A_{2}, B_{2}, G_{2}, A_{3}, B_{3}, C_{3}$. Another important result is that functions $c_{n_{i}}(\lambda)$ satisfying the system of generalized Riccati equations constructed from Riccatians of the type $\mathcal{L}_{r}$ exactly coincide with eigenvalues of the Gaudin spectral problem associated with algebra $\mathcal{L}_{r}$. This result suggests that the generalized Gaudin models admit a total separation of variables.
\end{abstract}

1. Introduction. The differential equations with large internal symmetries always have a great theoretical significance and, as a rule, admit many interesting mathematical

1991 Mathematics Subject Classification: Primary 34L40, Secondary 35P05, 81Q05.

This work was partially supported by the State Committee for Scientific Research (KBN) grant no. 2P30221706p01.

$\left({ }^{1}\right)$ The exact meaning of the adjective "spectral" will be clarified in subsection 1.1. Here we only note that the class of ordinary spectral Riccati equations contains, for example, the delinearized version of Lame equation.

The paper is in final form and no version of it will be published elsewhere. 
and physical applications.

One of such equations is the ordinary spectral Riccati equation which is very well known to theoretical physicists interested in properties of completely integrable quantum systems and their solutions. The main feature of this equation is that its solutions exactly coincide with Bethe ansatz solutions of completely integrable quantum Gaudin models associated with algebra $s l(2)$.

The aim of the present paper is to demonstrate that the ordinary spectral Riccati equation admits a very natural generalization to a multi-component case when instead of a single first-order nonlinear differential equation one considers the systems of nonlinear equations of higher orders. It turns out that solutions of these systems exactly coincide with Bethe ansatz solutions of generalized Gaudin problems associated with various simple Lie algebras.

The paper is organized as follows. Here, in introducion, we consider the simplest Riccati spectral problem (subsection 1.1) and simplest Gaudin spectral problem associated with algebra $s l(2)$ (subsection 1.2). In subsection 1.3 we demonstrate the coincidence of solutions of these problems and present a standard explanation of this fact.

The main body of the paper is devoted to construction of generalized Riccati spectral equations and their solutions. In section 2 we introduce all necessary notions and notations and formulate some general theorems. In particular, we introduce a new very important notion of Riccatians which play the role of elementary building blocks by constructing generalized Riccati equations. In section 3 we discuss the methods for calculating simplest Riccatians. The results of these calculations are collected in next section 4 . In section 5 we demonstrate remarkable parallels between systems of Riccatians and systems of independent Casimir invariants for simple Lie algebras. In the same section we give the final form of solution of the generalized Riccati spectral problem. In section 6 we consider the generalized Gaudin models associated with arbitrary simple Lie algebras and present their Bethe ansatz solutions. In last section 7 we demonstrate the coincidence of solutions of Riccati and Gaudin spectral problems and discuss possible ways of its explanation.

1.1 Simplest Riccati spectral problem. Consider the followig formal relation

$$
z^{\prime}(\lambda)+z^{2}(\lambda)=c(\lambda)
$$

in which $z(\lambda)$ and $c(\lambda)$ are assumed to be some analytic functions of a complex variable $\lambda$.

First of all note that the relation (1.1) (if one considers it as an equation) admits at least two interpretations:

1. Function $z(\lambda)$ is given, and function $c(\lambda)$ is being sought. This problem is trivial and has a unique solution.

2. Function $c(\lambda)$ is given, and function $z(\lambda)$ is being sought. This problem is nothing else than the ordinary Riccati equation. It has a one-parameter set of solutions. Except some very special cases this problem cannot be solved in quadratures [Korn and Korn 1971].

It turns out however that along with these two polar interpretations, there exists an interesting intermediate one which leads to a very rich set of solutions and has a great theoretical importance. Roughly speaking, the idea of this interpretation is to fix appro- 
priately some parts of both functions $z(\lambda)$ and $c(\lambda)$ and state the problem of finding the remaining parts of these functions. Before giving a rigorous formulation of this problem, let us introduce some necessary notions and notations.

Let $\mathcal{R}$ be the class of all rational functions $r(\lambda)$ of a single complex variable $\lambda$. This class can obviously be viewed as an infinite-dimensional linear vector space with a basis consisting of the so-called elementary rational functions. We denote these functions by $r_{a}^{n}(\lambda)$ and define them as $(\lambda-a)^{-n}$, for $a \neq \infty$, and as $\lambda^{n-1}$ for $a=\infty$. In both cases $n$ is a natural number.

We call a rational function singular (regular) at the point $\lambda=a$ if its expansion in elementary rational functions contains (does not contain) a term proportional to $r_{a}^{n}(\lambda)$ with some $n$. For example, according to our definition, function $r(\lambda)=\lambda^{-1}$ is singular at the point $\lambda=0$ and regular at all other points including infinity. As to the function $r(\lambda)=1$, it is singular at infinity but regular at all finite points.

Let $A$ be the a finite set of nonequal fixed complex numbers (one of which may be the infinity), and $B$ be the set of all the remaining numbers. Denote by $\mathcal{R}_{A}$ and $\mathcal{R}_{B}$ the classes of those rational functions from $\mathcal{R}$ whose singularities belong only to the sets $A$ and $B$, respectively. Considering the classes $\mathcal{R}_{A}$ and $\mathcal{R}_{B}$ as linear vector spaces we can write $\mathcal{R}_{A} \oplus \mathcal{R}_{B}=\mathcal{R}$. Denote also by $\mathcal{P}_{A}$ and $\mathcal{P}_{B}$ the projectors from $\mathcal{R}$ onto $\mathcal{R}_{A}$ and $\mathcal{R}_{B}$ for which we obviously have $\mathcal{P}_{A}+\mathcal{P}_{B}=1$.

Now we are ready to give a strict formulation of the problem of our interest.

Problem 1.1. Find all functions $z(\lambda), c(\lambda) \in \mathcal{R}$ satisfying the relation (1.1) under two additional constraints:

$$
\mathcal{P}_{A} z(\lambda)=F(\lambda), \quad \mathcal{P}_{B} c(\lambda)=0,
$$

in which $F(\lambda) \in \mathcal{R}_{A}$ is a given function.

The first constraint means that all the functions $z(\lambda)$ should have the same fixed projection $F(\lambda)$ onto the space $\mathcal{R}_{A}$ and, in principle, may have arbitrary singularities outside the set $A$. The second constraint means however that not any singularities of functions $z(\lambda)$ lying outside the set $A$ are admissible, but only those, at which the second function $c(\lambda)$ is regular.

Definition 1.1. The expression $P[z(\lambda)] \equiv z^{\prime}(\lambda)+z^{2}(\lambda)$ standing in the left hand side of formula (1.1) we shall call the Riccati polynomial of a functional variable $z(\lambda)$. Functions $z(\lambda)$ and $c(\lambda)$ satisfying the conditions of the problem 1.1 we shall call the eigenfunctions and eigenvalues of the Riccati polynomial $R[z(\lambda)]$. The set $\{z(\lambda), c(\lambda)\}$ of all such eigenfunctions and eigenvalues we shall call the spectrum of the Riccati polynomial, and the problem 1.1 itself - the Riccati spectral problem.

In order to find the general solution of this problem, it is reasonable to consider first the following auxiliary subproblem. Assume that the rational function $z(\lambda)$ has an isolated singularity at the point $\xi \notin A$ and try to find conditions under which the polynomial $R[z(\lambda)]$ is regular at the point $\lambda=\xi$. 
First of all note that the only possible singularity of the function $z(\lambda)$ at the point $\lambda=\xi$ may be simple pole because, as it can be easily verified, the higher poles cannot be cancelled in the expression for $R[z(\lambda)]$ at all. This allows one to represent function $z(\lambda)$ in the form

$$
z(\lambda)=y(\lambda)+\frac{v}{\lambda-\xi},
$$

where $v$ is some complex number and $y(\lambda)$ is a function regular at $\lambda=\xi$. Substituting (1.3) into Riccati polynomial $R[z(\lambda)]$ we obtain

$$
R\left[y(\lambda)+\frac{v}{\lambda-\xi}\right]=\frac{R_{0}[v, y(\xi)]}{(\lambda-\xi)^{2}}+\frac{R_{1}[v, y(\xi)]}{\lambda-\xi}+\text { regular terms. }
$$

The coefficients $R_{0}[v, y(\xi)]=v^{2}-v$ and $R_{1}[v, y(\xi)]=2 v y(\xi)$ we call the residues of the polynomial $R[z(\lambda)$. Now note that there exists a special value of $v$, namely $v=1$, for which both the residues become proportional to the function $y(\xi): R_{0}[1, y(\xi)]=0$ and $R_{0}[1, y(\xi)]=2 y(\xi)$. This means that for $v=1$ the condition of regularity of the polynomial $R[z(\lambda)]$ at the point $\lambda=\xi$ can be written as

$$
y(\xi)=0 .
$$

Formula (1.5) enables one to present the general solution of the problem 1.1. Indeed, from the condition of the absence of higher order poles in $z(\lambda)$ it follows that the most general form of this function is

$$
z(\lambda)=F(\lambda)+\sum_{i=1}^{M} \frac{v_{i}}{\lambda-\xi_{i}}
$$

where $M$ is an arbitrarily fixed non-negative integer and $\xi_{i}$ are some unknown parameters. Rewritting (1.6) in one of the following $M$ forms

$$
z(\lambda)=y_{i}(\lambda)+\frac{v_{i}}{\lambda-\xi_{i}}, \quad i=1, \ldots, M
$$

and using (1.5) we can conclude that the conditions of regularity of the function $R[z(\lambda)]$ at the points $\lambda=\xi_{i}$ are

$$
y_{i}\left(\xi_{i}\right)=0, \quad i=1, \ldots, M,
$$

provided that $v_{i}=1, i=1, \ldots, M$. Using the explicit form of fuctions $y_{i}(\lambda)$,

$$
y_{i}(\lambda)=F(\lambda)+\sum_{k=1, k \neq i}^{M} \frac{v_{k}}{\lambda-\xi_{k}}, \quad i=1, \ldots, M,
$$

we can write down the final solution of the problem 1.1 .

THEOREM 1.1. The most general solution of the Problem 1.1 has the following form

$$
\begin{gathered}
z(\lambda)=F(\lambda)+\sum_{i=1}^{M} \frac{1}{\lambda-\xi_{i}} \\
c(\lambda)=\left(F(\lambda)+\sum_{i=1}^{M} \frac{1}{\lambda-\xi_{i}}\right)^{\prime}+\left(F(\lambda)+\sum_{i=1}^{M} \frac{1}{\lambda-\xi_{i}}\right)^{2},
\end{gathered}
$$


where $M$ is an arbitrary non-negative integer and the numbers $\xi_{i}, i=1, \ldots, M$ satisfy the system of equations

$$
\sum_{k=1, k \neq i}^{M} \frac{1}{\xi_{i}-\xi_{k}}+F\left(\xi_{i}\right)=0, \quad i=1, \ldots, M
$$

For any $M=0,1, \ldots$ the system (1.10) has a finite set of solutions. Therefore the spectrum of the Riccati polynomial (1.1) is infinite and discrete.

The most interesting feature of these solutions is that their exactly coincide with Bethe ansatz solutions of the so-called $s l(2)$ Gaudin spectral problem. But before demonstrating this fact, it is reasonable to remind the reader what does the $s l(2)$ Gaudin problem mean.

1.2. Simplest Gaudin spectral problem. The Gaudin spectral problems can be formulated in terms of the so-called Gaudin algebras which are some special infinite-dimensional extensions of simple Lie algebras. In this section we consider the simplest Gaudin algebra $\mathcal{G}[s l(2)]$ associated with algebra $\operatorname{sl}(2)$. The discussion of the general case will be given in section 6 .

The three generators of the Gaudin algebra $\mathcal{G}[s l(2)]$, which we denote by $S^{ \pm}(\lambda)$ and $S(\lambda)$, are parametrized by a complex parameter $\lambda$ playing the role of an additional continuous index. The commutation relations for these generators have the form

$$
\left[S(\lambda), S^{ \pm}(\mu)\right]= \pm \frac{S^{ \pm}(\lambda)-S^{ \pm}(\mu)}{\mu-\lambda},\left[S^{+}(\lambda), S^{-}(\mu)\right]=\frac{S(\lambda)-S(\mu)}{\mu-\lambda},
$$

and can be viewed as generalizations of commutation relations for algebra $s l(2)$ [Gaudin 1976, 1983].

The lowest weight representations of Gaudin algebra $\mathcal{G}[s l(2)]$ can be defined by the formulas

$$
S^{-}(\lambda)|0\rangle=0, \quad S(\lambda)|0\rangle=F(\lambda)|0\rangle .
$$

As in the $s l(2)$ case, $|0\rangle$ is the lowest weight vector, and $F(\lambda)$ is the corresponding lowest weight which, however, is here a function rather than a constant. The representation space is given by the formula

$$
W_{F(\lambda)}=\text { linear span of vectors }\left\{S^{+}\left(\lambda_{1}\right) \cdots S^{+}\left(\lambda_{n}\right)|0\rangle\right\}, n=0,1,2, \ldots
$$

with arbitrary $\lambda_{1}, \ldots, \lambda_{n}$. Generally, this space is infinite-dimensional.

Consider the operator

$$
C(\lambda)=S^{2}(\lambda)+S^{+}(\lambda) S^{-}(\lambda)+S^{-}(\lambda) S^{+}(\lambda),
$$

which belongs to the universal enveloping algebra of algebra $\mathcal{G}[\operatorname{sl}(2)]$ and has the form similar to the form of the Casimir operator for algebra $\operatorname{sl}(2)$. For this reason we call (1.15) the Casimir-Gaudin operator. It is not difficult to see, however, that this operator is not a Casimir invariant for the algebra $\mathcal{G}[s l(2)]$ because it explicitly depends on the continuous index $\lambda$ which is not contracted. In fact, one can check that $C(\lambda)$ does not commute with the generators of Gaudin algebra. At the same time, it has another remarkable property which is the commutativity of its "values" for different values of $\lambda$ :

$$
[C(\lambda), C(\mu)]=0 .
$$


This property suggests to interpret $C(\lambda)$ as a generating function of commuting integrals of motion (hamiltonians) for some quantum system. It can be shown that this system is completely integrable in the sense that it admits enough number of independent $\left({ }^{2}\right)$ commuting integrals of motion [Sklyanin 1991].

It turns out that this system is not only completely integrable, but also exactly solvable $\left(^{3}\right)$. Considering the representation space of Gaudin algebra $\mathcal{G}[\operatorname{sl}(2)]$ as a space of states, we can formulate the following analog of the Schrödinger problem.

Problem 1.2. Find all solutions of the spectral equation

$$
C(\lambda) \phi=c(\lambda) \phi, \quad \phi \in W_{F(\lambda)},
$$

provided that the lowest weight $F(\lambda)$ is given.

Definition 1.2. The equation (1.17) is called the $s l(2)$ Gaudin spectral equation and the models described by "hamiltonians" $C(\lambda)$ we refer to as $\operatorname{sl}(2)$ Gaudin models.

Theorem 1.2. An explicit solution of the Problem 1.2 does exist and has an elegant and purely algebraic form:

$$
\begin{gathered}
\phi=S^{+}\left(\xi_{1}\right) \cdot \ldots \cdot S^{+}\left(\xi_{M}\right)|0\rangle \\
c(\lambda)=\left(F(\lambda)+\sum_{i=1}^{M} \frac{1}{\lambda-\xi_{i}}\right)^{\prime}+\left(F(\lambda)+\sum_{i=1}^{M} \frac{1}{\lambda-\xi_{i}}\right)^{2} .
\end{gathered}
$$

Here $M$ is an arbitrary non-negative integer and the numbers $\xi_{1}, \ldots, \xi_{M}$ satisfy the system of equations

$$
\sum_{k=1, k \neq i}^{M} \frac{1}{\xi_{i}-\xi_{k}}+F\left(\xi_{i}\right)=0, \quad i=1, \ldots, M
$$

For any rational function $F(\lambda)$ and for any finite $M$ the set of equation (1.19) is finite. This means that the whole spectrum of the sl(2) Gaudin model is infinite and discrete.

The substitution (1.18a) solving the problem is called the Bethe ansatz, and the equations (1.19) are known under generic name of Bethe ansatz equations. The action of the operator $C(\lambda)$ on the trial Bethe vector (1.18a) leads to two groups of terms, one of which are proportional to (1.18a) and other are not. The latter are often called the unvanted terms, and the equations (1.19) are exactly the conditions for their cancellation. The complete proof of theorem 1.2 can be found in [Gaudin 1983].

1.3. On equivalence of Riccati and Gaudin problems in the simplest case. Comparing formulas (1.10b) and (1.11) with (1.18b) and (1.19), the reader can easily make sure that solutions of Riccati and Gaudin spectral problems exactly coincide! This suggest that there should be some deep relationship between these problems. Actually, it turns out that

$\left({ }^{2}\right)$ The independence of quantum integrals of motion is understood here as functional independence of their classical analogs obtained after dequantization.

$\left({ }^{3}\right)$ The notions of complete integrability and exact solvability in quantum mechanics do not necessarily coincide [Doebner and Ushveridze 1994]. 
the coincidence of solutions of problems 1.1 and 1.2 is not accidental and the reason for it is the separability of variables in the Gaudin spectral equation [Sklyanin 1987]. Moreover, there exists a procedure (which is known in the literature as the inverse procedure of separation of variables which enables one to derive the Gaudin spectral problem from the Riccati one [Ushveridze 1989, 1994]. The main idea of this derivation is based on the fact that the Riccati equation is linearizable by the substitution $z(\lambda)=\psi^{\prime}(\lambda) / \psi(\lambda)$ after which it takes the form of the so-called linear multi-parameter spectral equations, i.e. linear spectral equations containing many spectral parameters. The number of spectral parameters is finite if $F(\lambda)$ is a rational function. Moreover, these parameters enter into equation linearly. It is known that any linear multi-parameter spectral equation with linear dependence on spectral parameters can be interpreted as an equation appearing after separation of variables in some multi-dimensional completely integrable quantum system. The explicit construction of this system shows that it is nothing else than the $s l(2)$ Gaudin model.

We do not intend to discuss in this paper the details of this derivation because it can be found in references [Ushveridze 1989, 1994]. We aimed only to stress the fact that equation (1.1) contains in a hidden form the complete information of the $\operatorname{sl}(2)$ Gaudin model and its solutions.

In next sections of this paper we construct the generalizations of equation (1.1) which will contain all information of general Gaudin models (associated with arbitrary simple Lie algebras) and their solutions.

\section{Generalized Riccati spectral problem}

2.1. Preliminaries. In this section we shall consider various differential operators acting on functions of a complex variable $\lambda$. The simplest operator of such sort is the ordinary first-order differential operator $\partial \equiv \partial / \partial \lambda$. We shall consider it as a graded object, having, by definition, the unit degree of homogeneity,

$$
\operatorname{deg} \partial=1
$$

The functions on which the differential operators will act, will be vector-valued complex analytic functions $\mathbf{z}(\lambda)=\left\{z_{1}(\lambda), \ldots, z_{r}(\lambda)\right\}$. These functions also will be considered as graded objects, having, by definition, a vector grading $\mathbf{n}=\left\{n_{1}, \ldots, n_{r}\right\}$. We express this fact by writing $\operatorname{deg} z_{1}(\lambda)=n_{1}, \ldots, \operatorname{deg} z_{r}(\lambda)=n_{r}$, or, in vector notations,

$$
\operatorname{deg} \mathbf{z}(\lambda) \equiv \mathbf{n} \text {. }
$$

Below we shall always assume that the components of vector $\mathbf{n}$ are natural numbers ordered as $n_{1} \leq \ldots \leq n_{r}$. We can also establish some order relations between different vectors by writing $\mathbf{n}_{1} \leq \mathbf{n}_{2}$ if each component of $\mathbf{n}_{1}$ is equal or less than the corresponding component of $\mathbf{n}_{2}$. In this sense, there exist a minamal vector consisting of unit components only. We denote it by $\mathbf{u}=\{1, \ldots, 1\}$. This vector will not be used only as a grading vector but also as a tool for simplification of various notations. So, for example, the sum of all components of vector $\mathbf{n}$ we shall often write in compact form as $\mathbf{u} \cdot \mathbf{n}$.

Let $\Phi(\mathbf{n})$ denote a space of graded vector-valued complex analytic functions $\mathbf{z}(\lambda)$ satisfying the condition (2.2). Taking into account formula (2.1), we can formulate several 
obvious statements:

1. Let $\mathbf{z}(\lambda) \in \Phi(\mathbf{n})$. Then $\partial \mathbf{z}(\lambda) \in \Phi(\mathbf{n}+\mathbf{u})$.

2. Let $\mathbf{z}_{1}(\lambda), \mathbf{z}_{2}(\lambda) \in \Phi(\mathbf{n})$ and $c_{1}, c_{2} \in C$. Then $c_{1} \mathbf{z}_{1}(\lambda)+c_{2} \mathbf{z}_{2}(\lambda) \in \Phi(\mathbf{n})$.

3. Let $\mathbf{z}_{1}(\lambda) \in \Phi\left(\mathbf{n}_{1}\right)$ and $\mathbf{z}_{2}(\lambda) \in \Phi\left(\mathbf{n}_{2}\right)$. Then $\mathbf{z}_{1}(\lambda) \otimes \mathbf{z}_{2}(\lambda) \in \Phi\left(\mathbf{n}_{1} \otimes \mathbf{n}_{2}\right)$.

4. Let $\mathbf{z}_{1}(\lambda) \in \Phi\left(\mathbf{n}_{1}\right)$ and $\mathbf{z}_{2}(\lambda) \in \Phi\left(\mathbf{n}_{2}\right)$. Then $\mathbf{z}_{1}(\lambda) \oplus \mathbf{z}_{2}(\lambda) \in \Phi\left(\mathbf{n}_{1} \oplus \mathbf{n}_{2}\right)$.

5. Let $\mathbf{z}(\lambda) \in \Phi(\mathbf{n})$, where $\left.\mathbf{n}=\mathbf{n}_{1} \oplus \mathbf{n}_{2}\right)$. Then $\mathbf{z}(\lambda)=\mathbf{z}_{1}(\lambda) \oplus \mathbf{z}_{2}(\lambda)$, where $\mathbf{z}_{1}(\lambda) \in$ $\Phi\left(\mathbf{n}_{1}\right)$ and $\mathbf{z}_{2}(\lambda) \in \Phi\left(\mathbf{n}_{2}\right)$.

Using these simple rules and starting with the elements $\mathbf{z}_{1}(\lambda)$ of the space $\Phi\left(\mathbf{n}_{1}\right)$, we can construct the elements $\mathbf{z}_{2}(\lambda)$ of another space $\Phi\left(\mathbf{n}_{2}\right)$. In this case the components of vectors $\mathbf{z}_{2}(\lambda)$ will have the form of polynomials in components of vectors $\mathbf{z}_{1}(\lambda)$ and their finite derivatives.

Definition 2.1. The non-linear differential operators $P$ constructed according the rules $1-5$ and realizing the mapping $\Phi\left(\mathbf{n}_{1}\right) \rightarrow \Phi\left(\mathbf{n}_{2}\right)$ with $\operatorname{dim} \mathbf{n}_{1}=\operatorname{dim} \mathbf{n}_{2}=r$, we shall call the r-operators of the type $\left|\mathbf{n}_{2}\right\rangle\left\langle\mathbf{n}_{1}\right|$.

Let $P_{1}$ and $P_{2}$ be two r-operators of the types $\left|\mathbf{n}_{1}\right\rangle\left\langle\mathbf{m}_{1}\right|$ and $\left|\mathbf{n}_{2}\right\rangle\left\langle\mathbf{m}_{2}\right|$, respectively. We call $P_{1}$ compatible with $P_{2}$ if $\mathbf{n}_{1}=\mathbf{m}_{2}$. In this case, it is possible to construct a composite r-operator $P_{2} \circ P_{1}$ of the type $\left|\mathbf{n}_{2}\right\rangle\left\langle\mathbf{m}_{1}\right|$.

Let us now introduce an important notion of r-determinants. Consider a transformation of elements $\mathbf{z}_{1}(\lambda) \in \Phi\left(\mathbf{n}_{1}\right)$ into elements $\mathbf{z}_{2}(\lambda) \in \Phi\left(\mathbf{n}_{2}\right)$ realized by a certain r-operator $P$ :

$$
\mathbf{z}_{2}(\lambda)=P\left[\mathbf{z}_{1}(\lambda)\right]
$$

Let $\hat{\mathbf{z}}_{1}(\lambda)=\left\{\partial^{k} \mathbf{z}_{1}(\lambda)\right\}_{k=1}^{\infty}$ and $\hat{\mathbf{z}}_{2}(\lambda)=\left\{\partial^{k} \mathbf{z}_{2}(\lambda)\right\}_{k=1}^{\infty}$ denote some infinite-dimensional vector functions of $\lambda$. Acting on both hand sides of (2.3) by the operators $\partial^{k}$ with $k=$ $0,1,2, \ldots, \infty$, one can construct a new infinite-dimensional and non-differential operator $\hat{P}$ realizing the transformation of vectors $\hat{\mathbf{z}}_{1}(\lambda)$ into vectors $\hat{\mathbf{z}}_{2}(\lambda)$ :

$$
\hat{\mathbf{z}}_{2}(\lambda)=\hat{P}\left[\hat{\mathbf{z}}_{1}(\lambda)\right] .
$$

Definition 2.2. The Jacobian of the transformation (2.4) will be called the $r$ determinant of the r-operator $P$ and denoted by r-det $P$.

Lemma 2.1. The r-determinants have all properties of ordinary determinants. In particular,

$$
r-\operatorname{det}\left(P_{2} \circ P_{1}\right)=\left(r-\operatorname{det} P_{2}\right) \cdot\left(r-\operatorname{det} P_{1}\right),
$$

for any r-operators $P_{1}$ and $P_{2}$.

Definition 2.3. We call a r-operator $P$ degenerate if $r-\operatorname{det} P=0$, and nondegenerate if $r-\operatorname{det} P \neq 0$.

Lemma 2.2. Let $P$ be a r-operator of the type $|\mathbf{n}\rangle\langle\mathbf{m}|$. If $P$ is non-degenerate, then $\mathbf{n} \geq \mathbf{m}$.

Definition 2.4. The non-degenerate r-operators of the type $|\mathbf{n}\rangle\langle\mathbf{n}|$ we shall call pseudo-diagonal r-operators. 
2.2. Riccati operators and polynomials. Riccati operators are important particular cases of general r-operators. Below we give their definition and discuss some important properties.

Definition 2.5. The non-degenerate r-operator of the type $|\mathbf{n}\rangle\langle\mathbf{u}|$ we shall call the Riccati operator of dimension $r=\operatorname{dim} \mathbf{u}$ and age $|\mathbf{n}\rangle$. Let $R_{1}$ and $R_{2}$ be two Riccati operators of ages $\left|\mathbf{n}_{1}\right\rangle$ and $\left|\mathbf{n}_{2}\right\rangle$. We call the operator $R_{1}$ older (not younger) than $R_{2}$ or youger (not older) than $R_{2}$ if $\mathbf{n}_{1}>\mathbf{n}_{2}\left(\mathbf{n}_{1} \geq \mathbf{n}_{2}\right)$ or $\mathbf{n}_{1}<\mathbf{n}_{2}\left(\mathbf{n}_{1} \leq \mathbf{n}_{2}\right)$, respectively.

Any Riccati operator $R^{0}$ of age $\left|\mathbf{n}^{0}\right\rangle$ is compatible with any r-operator $P$ of age $|\mathbf{n}\rangle\left\langle\mathbf{n}^{0}\right|$. If $P$ is non-degenerate, then the composite operator $R=P \circ R$ is again a Riccati operator of age $|\mathbf{m}\rangle$. According to lemma 2.2, the operator $R$ is always not younger than $R^{0}$. Note also that pseudo-diagonal transformations conserve the age of Riccati operators. The Riccati operators connected by some pseudo-diagonal transformation we shall call age-equivalent.

Definition 2.6. Let $R$ be a Riccati operator of dimension $r$ and age $|\mathbf{n}\rangle$ acting in the space $\Phi(\mathbf{u})$. If $\mathbf{z}(\lambda) \in \Phi(\mathbf{u})$, then $R[\mathbf{z}(\lambda)] \in \Phi(\mathbf{n})$ is a $r$-component vector function. Its components, which are the homogeneous polynomials in components of function $\mathbf{z}(\lambda)$ and their finite derivatives, we shall denote by $R_{n_{i}}[\mathbf{z}(\lambda)], i=1, \ldots, r$ and call the Riccati polynomials of degrees $n_{i}, i=1, \ldots, r$.

As an example, below we present the most general form of Riccati polynomials of degrees $n=0,1,2,3$ and 4 .

$$
\begin{gathered}
R_{0}[\mathbf{z}(\lambda)]=A_{0} \\
R_{1}[\mathbf{z}(\lambda)]=A_{1} \cdot \mathbf{z}(\lambda) \\
R_{3}[\mathbf{z}(\lambda)]=A_{3} \cdot \partial^{2} \mathbf{z}(\lambda)+B_{3} \cdot \partial \mathbf{z}(\lambda) \otimes \mathbf{z}(\lambda)+C_{3} \cdot \mathbf{z}(\lambda) \otimes \mathbf{z}(\lambda) \otimes \mathbf{z}(\lambda), \\
R_{4}[\mathbf{z}(\lambda)]=A_{4} \cdot \partial^{3} \mathbf{z}(\lambda)+B_{4} \cdot \partial^{2} \mathbf{z}(\lambda) \otimes \mathbf{z}(\lambda) \\
+C_{4} \cdot \partial \mathbf{z}(\lambda) \otimes \partial \mathbf{z}(\lambda)+D_{4} \cdot \partial \mathbf{z}(\lambda) \otimes \mathbf{z}(\lambda) \otimes \mathbf{z}(\lambda) \\
+E_{4} \cdot \mathbf{z}(\lambda) \otimes \mathbf{z}(\lambda) \otimes \mathbf{z}(\lambda) \otimes \mathbf{z}(\lambda) .
\end{gathered}
$$

Here " $\otimes$ " means the ordinary tensor product and "." denotes contraction of tensors of equal rank. $A_{n}, B_{n}, C_{n}, D_{n}$ and $E_{n}$ are constant tensors of rank $n$.

2.3. Riccati spectral problem. Now we can start the discussion of spectral properties of Riccati operators. Let $\mathbf{z}(\lambda) \in \Phi(\mathbf{u})$ and $\mathbf{c}(\lambda) \in \Phi(\mathbf{n})$ be two analytic functions satisfying the following relation:

$$
R[\mathbf{z}(\lambda)]=\mathbf{c}(\lambda),
$$

where $R$ is a certain Riccati operator of age $|\mathbf{n}\rangle$. This relation can be interpreted as a generalization of the relation (1.1).

As in the case of (1.1) the relation (2.3) (if one considers it as an equation) admits two standard interpretations:

1. Functions $\mathbf{z}(\lambda)$ are given, and functions $\mathbf{c}(\lambda)$ are being sought. This problem is trivial and has a unique solution. 
2. Functions $\mathbf{c}(\lambda)$ are given, and functions $\mathbf{z}(\lambda)$ are being sought. This problem can be considered as a generalization of the ordinary Riccati equation. It has a $\mathbf{u} \cdot(\mathbf{n}-\mathbf{u})$ parameter set of solutions. Generally, this problem cannot be solved in quadratures.

In this section we discuss the third (intermediate) case which is realized when some parts of both sets of functions $\mathbf{z}(\lambda)$ and $\mathbf{c}(\lambda)$ are simultaneously given and the remaining parts of these functions are being sought. It is reasonable to change a little bit the meaning of notations introduced in section 1 . Hereafter we denote by $\mathcal{R}$ the class of all $r$-component vector-valued rational functions of a single complex variable $\lambda$. We denote also by $\mathcal{R}_{A}$ and $\mathcal{R}_{B}$ the classes of those rational functions from $\mathcal{R}$ whose singularities belong only to the sets $A$ and $B$ defined in section 1. As before, $\mathcal{P}_{A}$ and $\mathcal{P}_{B}$ will be the projectors from $\mathcal{R}$ onto $\mathcal{R}_{A}$ and $\mathcal{R}_{B}$. Then the problem of our interest can be formulated as follows.

Problem 2.1. Find all functions $\mathbf{z}(\lambda), \mathbf{c}(\lambda) \in \mathcal{R}$ satisfying the relation (2.3) under two additional constraints:

$$
\mathcal{P}_{A} \mathbf{z}(\lambda)=\mathbf{F}(\lambda), \quad \mathcal{P}_{B} \mathbf{c}(\lambda)=0,
$$

in which $\mathbf{F}(\lambda) \in \mathcal{R}_{A}$ is a given vector function.

Definition 2.7. Functions $\mathbf{z}(\lambda)$ and $\mathbf{c}(\lambda)$ satisfying the conditions of the problem we shall call the eigenfunctions and eigenvalues of the Riccati operator $R$. The set $\{\mathbf{z}(\lambda), \mathbf{c}(\lambda)\}$ of all such eigenfunctions and eigenvalues we shall call the spectrum of the Riccati operator $R$, and the problem 2.1 itself we call the (generalized) Riccati spectral problem.

Below we will have many opportunities to demonstrate that not for any Riccati operator the solvability of the problem 2.1 can be guaranteed.

Definition 2.8. We call a Riccati operator admissible if its spectrum is non-empty.

The following problem immediately arises:

Problem 2.2. Find all admissible Riccati operators.

2.4. Residues and regularizators. In order to solve the problem 2.2 in full generality, it is reasonable to choose the same strategy as in section 1 and consider first the following auxiliary subproblem.

Assume that the rational function $\mathbf{z}(\lambda)$ has an isolated singularity at the point $\xi \notin A$ and try to find conditions under which the function $\mathbf{c}(\lambda)=R[\mathbf{z}(\lambda)]$ is regular at the point $\lambda=\xi$.

It is easy to check that the only possible singularity of the function $\mathbf{z}(\lambda)$ at the point $\lambda=\xi$ may be a simple pole because the higher poles cannot be cancelled in the expression for $\mathbf{c}(\lambda)$ at all. This allows one to represent function $\mathbf{z}(\lambda)$ in the form

$$
\mathbf{z}(\lambda)=\mathbf{y}(\lambda)+\frac{\mathbf{v}}{\lambda-\xi},
$$

where $\mathbf{v}$ is some complex vector and $\mathbf{y}(\lambda)$ is a vector-valued function regular at $\lambda=\xi$. Acting on (2.9) by a certain Riccati operator $R$, we obtain a vector-valued function whose 
components can be represented in the form:

$$
R_{n_{i}}\left[\mathbf{y}(\lambda)+\frac{\mathbf{v}}{\lambda-\xi}\right]=\sum_{k=0}^{n_{i}-1} \frac{R_{i k}[\mathbf{v}, \mathbf{y}(\xi)]}{(\lambda-\xi)^{n_{i}-k}}+\text { regular terms, } \quad i=1, \ldots, r .
$$

Here $R_{i k}[\mathbf{v}, \mathbf{y}(\xi)]$ are some vector functions of $\mathbf{v}$ and $\partial^{l} \mathbf{y}(\xi), l \geq 0$. It is not difficult to show that these functions are some Riccati polynomials of degrees $k=0,1, \ldots, n_{i}-1$. Indeed, for the equality $\operatorname{deg} \partial^{l} \mathbf{z}(\lambda)=l+1$ to be compatible with (2.9) it is necessary to take $\operatorname{deg} \mathbf{y}(\lambda)=\mathbf{u}$ and $\operatorname{deg}(\lambda-\xi)=-1$, provided that $\operatorname{deg} \mathbf{v}=0$. The fact that both hand sides of (2.10) should have the same degrees $n_{i}$ implies that

$$
\operatorname{deg} R_{i k}[\mathbf{v}, \mathbf{y}(\xi)]=k .
$$

Definition 2.9. The polynomials $R_{i k}[\mathbf{v}, \mathbf{y}(\xi)]$ of degrees $k=0, \ldots, n_{i_{1}}$ playing the role of the coefficients for the singular terms in the expansion (2.6), we shall call the residues of the the Riccati operator $R$.

From formula (2.10) it follows that the condition of regularity of function $\mathbf{c}(\lambda)$ ] at the point $\lambda=\xi$ is the condition of a simultaneous vanishing of all the residues of $R$ :

$$
R_{i k}[\mathbf{v}, \mathbf{y}(\xi)]=0, \quad k=0,1, \ldots, n_{i}-1, \quad i=1, \ldots, r .
$$

The total number of these conditions, equal to the number of all residues, is obviously $\mathbf{u} \cdot \mathbf{n}$, while the number of unknowns in (2.8), consisting of the components of vector $\mathbf{v}$ and parameter $\xi$, is $\operatorname{dim} \mathbf{u}+1$. In all the cases when $\mathbf{i} \cdot \mathbf{n}>\operatorname{dim} \mathbf{u}+1$ the system $(2.12)$ is over-determined and thus has no solutions at all.

Assume, however, that there exist some special values of $\mathbf{v}$ for which all $\mathbf{u} \cdot \mathbf{n}$ residues of $R$ become proportional to a single Riccati polynomial of degree $l$ :

$$
R_{i k}[\mathbf{v}, \mathbf{y}(\xi)]=Q_{l}[\mathbf{y}(\xi)] R_{i, k-l}^{\prime}[\mathbf{v}, \mathbf{y}(\xi)], \quad k=0,1, \ldots, n_{i}-1, \quad i=1, \ldots, r .
$$

In this case the system of conditions $(2.12)$ can be reduced to a single equation

$$
Q_{l}[\mathbf{y}(\xi)]=0
$$

for a single unknown $\xi$. Obviously, in general case such systems are solvable.

Definition 2.10. The vector $\mathbf{v}$ for which the residues $R_{i k}[\mathbf{v}, \mathbf{y}(\xi)]$ of a Riccati operator $R$ become divisible (without remainder) by a certain Riccati polynomial $Q_{l}[\mathbf{y}(\xi)]$ of degree $l$, we call the $l$-regularizing vector, and the polynomial $Q_{l}[\mathbf{y}(\xi)]$ itself we call the l-regularizing polynomial. The set of pairs $\left\{\mathbf{v}, Q_{l}[\mathbf{y}(\xi)]\right\}$ consisting of all $l$-regularizing vectors and corresponding $l$-regularizing polynomials, we call the l-regularizator of the Riccati operator $R$ and denote it by reg $R$. The Riccati operators with non-empty $l$ regularizators we call $l$-regularizable.

Conjecture 2.1. For any Riccati operator of dimension $r$, the l-regularizator consists of exactly $r$ elements.

At the present time we have no satisfactory proof of this conjecture, however, the experience accumulated by studying various concrete Riccati operators indicates that it should be true. At least it is true for all Riccati operators which we intend to discuss in the present paper. 
2.5. General theorem. Now we are ready to formulate the following theorem.

TheOREm 2.1. Let $R$ be a given Riccati operator of dimension $r$ with a non-empty reqularizator $\left\{\mathbf{v}^{a}, Q_{l}^{a}[\mathbf{y}(\xi)]\right\}, a=1, \ldots, r$. Then solution of the corresponding Riccati spectral problem 2.1 has the following form:

$$
\begin{gathered}
\mathbf{z}(\lambda)=\mathbf{F}(\lambda)+\sum_{a} \sum_{i=1}^{M_{a}} \frac{\mathbf{v}^{a}}{\lambda-\xi_{i}^{a}}, \\
\mathbf{c}(\lambda)=R\left[\mathbf{F}(\lambda)+\sum_{a} \sum_{i=1}^{M_{a}} \frac{\mathbf{v}^{a}}{\lambda-\xi_{i}^{a}}\right],
\end{gathered}
$$

where $M_{a}, a=1, \ldots, r$ are arbitrary non-negative integers, and the parameters $\xi_{i}^{a}, i=$ $1, \ldots, M_{a}, a=1, \ldots, r$ satisfy the system of equations

$$
Q_{l}^{a}\left[\mathbf{F}\left(\xi_{i}^{a}\right)+\sum_{b} \sum_{k=1}^{M^{b}} \frac{\mathbf{v}^{b}}{\xi_{i}^{a}-\xi_{k}^{b}}\right]=0, \quad i=1, \ldots, M_{a}, a=1, \ldots, r .
$$

For any set of non-negative integers $M_{a}, a=1, \ldots, r$ the equations (2.16) have a nonempty (finite) set of solutions, and thus, the Riccati operator under consideration is admissible and has an infinite and discrete spectrum.

Proof. The proof of this theorem is essentially the same as the proof of the Theorem 1.1 of Section 1. Indeed, using the same reasonongs as in Section 1, we can conclude that the most general form of function $\mathbf{z}(\lambda)$ satisfying the constraints (2.8) is (2.15a), where $M_{a}$ are some arbitrarily fixed non-negative integers, $\mathbf{v}^{a}$ are arbitrary vectors and $\xi_{i}^{a}$ are some complex parameters. Rewritting (2.17) in one of the following $M_{1}+\ldots+M_{r}$ forms

$$
\mathbf{z}(\lambda)=\mathbf{y}_{i}^{a}(\lambda)+\frac{\mathbf{v}_{i}^{a}}{\lambda-\xi_{i}^{a}}, \quad i=1, \ldots, M_{a}, \quad a=1, \ldots, r
$$

and using (2.14), we can conclude that the conditions of regularity of the function $\mathbf{c}(\lambda)$ ] at the points $\lambda=\xi_{i}^{a}$ are

$$
Q_{l}^{a}\left[\mathbf{y}_{i}^{a}\left(\xi_{i}^{a}\right)\right]=0, \quad i=1, \ldots, M_{a}, \quad a=1, \ldots, r,
$$

provided that $\mathbf{v}^{a}$ are regularizing vectors. Using the explicit form of functions $\mathbf{y}_{i}^{a}(\lambda)$,

$$
\mathbf{y}_{i}^{a}(\lambda)=\mathbf{F}(\lambda)+\sum_{b} \sum_{k=1}^{M^{b}} \frac{\mathbf{v}^{b}}{\lambda-\xi_{k}^{b}}, \quad i=1, \ldots, M_{a}, \quad a=1, \ldots, r,
$$

we can write down the final solution $(2.15),(2.16)$ of the Problem 2.1. This completes the proof of the theorem.

This theorem enables one to reduce the problem 2.2 of finding all admissible Riccati operators to a more simple and concrete one:

Problem 2.3. Find all $l$-regularizable Riccati operators and construct their $l$ regularizators.

Before trying to solve this problem it is useful to introduce a very important notion of Riccatians. This will be done in next subsection. 
2.6. Riccatians. We start with two simple lemmas which enable one to estimate the measure of ambigouity in fixing Riccati operators by their $l$-regularizators.

Lemma 2.1. Let $R^{0}$ be some l-regularizable Riccati operator of age $\left|\mathbf{n}^{0}\right\rangle$, compatible with a certain r-polynomial $P$ of type $|\mathbf{n}\rangle\left\langle\mathbf{n}^{0}\right|$. Then the composite Riccati operator $R=$ $P \circ R^{0}$ of age $|\mathbf{n}\rangle$ is also l-regularizable and reg $R=\operatorname{reg} R^{0}$.

P r o of. Let $\mathbf{v}$ and $Q_{l}[\mathbf{y}(\lambda)]$ be some $l$-regularizing vector and corresponding $l$-regularizing polynomial. Using formulas of the previous section, we can write

$$
R^{0}[\mathbf{z}(\lambda)]=R^{0}\left[\mathbf{y}(\lambda)+\frac{\mathbf{v}}{\lambda-\xi}\right]=Q_{l}[\mathbf{y}(\xi)] \cdot \mathbf{s}(\lambda)+\mathbf{r}(\lambda),
$$

where $\mathbf{s}(\lambda)$ and $r(\lambda)$ denote some functions of $\lambda$, which, respectively, are singular and regular at the point $\lambda=\xi$. Taking the derivative of the both hand sides of formula (2.20), we obtain

$$
\partial R^{0}[\mathbf{z}(\lambda)]=\partial R^{0}\left[\mathbf{y}(\lambda)+\frac{\mathbf{v}}{\lambda-\xi}\right]=Q_{l}[\mathbf{y}(\xi)] \cdot \partial \mathbf{s}(\lambda)+\partial \mathbf{r}(\lambda) .
$$

We see that the singular part of this expansion is also proportional to $Q_{l}[\mathbf{y}(\xi)]$.

Let now $R_{1}^{0}$ and $R_{2}^{0}$ be two Riccati operators for which $\mathbf{v}$ and $Q_{l}[\mathbf{y}(\xi)]$ are known to be $l$-regularizing vector and $l$-regularizing polynomial. Then we can write

$$
\begin{aligned}
& R_{1}^{0}[\mathbf{z}(\lambda)]=R_{1}^{0}\left[\mathbf{y}(\lambda)+\frac{\mathbf{v}}{\lambda-\xi}\right]=Q_{l}[\mathbf{y}(\xi)] \cdot \mathbf{s}_{1}(\lambda)+\mathbf{r}_{1}(\lambda), \\
& R_{2}^{0}[\mathbf{z}(\lambda)]=R_{2}^{0}\left[\mathbf{y}(\lambda)+\frac{\mathbf{v}}{\lambda-\xi}\right]=Q_{l}[\mathbf{y}(\xi)] \cdot \mathbf{s}_{2}(\lambda)+\mathbf{r}_{2}(\lambda),
\end{aligned}
$$

and, consequently,

$$
\begin{gathered}
R_{1}^{0}[\mathbf{z}(\lambda)] \otimes R_{2}^{0}[\mathbf{z}(\lambda)]=R_{1}^{0}\left[(\lambda)+\frac{\mathbf{v}}{\lambda-\xi}\right] \otimes R_{2}^{0}\left[(\lambda)+\frac{\mathbf{v}}{\lambda-\xi}\right]= \\
Q_{l}^{2}[\mathbf{y}(\xi)] \cdot \mathbf{s}_{1}(\lambda) \otimes \mathbf{s}_{2}(\lambda)+Q_{l}[\mathbf{y}(\xi)] \cdot\left(\mathbf{s}_{1}(\lambda) \otimes \mathbf{r}_{2}(\lambda)+\mathbf{r}_{1}(\lambda) \otimes \mathbf{s}_{2}(\lambda)\right)+\mathbf{r}_{1}(\lambda) \otimes \mathbf{r}_{2}(\lambda) .
\end{gathered}
$$

We see that the singular part of this expression is, as before, proportional to $Q_{l}[\mathbf{y}(\xi)]$. Because the construction of the Riccati operator $R$ implies the use of only two above operations of differentiation and multiplication, we can conclude that the singular part of the final expression for $R[\mathbf{z}(\lambda)]$ will again be proportional to $Q_{l}[\mathbf{y}(\lambda)]$. This means that the system of composite Riccati polynomials should have at least the same $l$-regularizator as the initial system. According to conjecture 2.1, these $l$-regularizators should coincide.

Lemma 3.1 demonstrates that $l$-regularizator does not fix a Riccati operator uniquely. There is always possible to start with a given Riccati operator and construct another Riccati operator with the same $l$-regularizator but having a greater age.

DEFinition 2.11. The Riccati operators, having the minimal age among all other Riccati operators with the same $l$-regularizator, we shall call minimal Riccati operators.

It is not difficult to see that minimal Riccati operators also cannot be uniquely determined by their $l$-regularizators, because of the existence of age conserving pseudo-diagonal transformations. 
LEMma 2.2. Let $R^{1}$ and $R^{2}$ be two l-regularizable Riccati operators. Let a new Riccati operator $R$ be defined by the formula

$$
R[\mathbf{z}(\lambda)]=R\left[\mathbf{z}^{1}(\lambda)\right] \oplus R^{2}\left[\mathbf{z}^{2}(\lambda)\right]
$$

in which $\mathbf{z}(\lambda)=\mathbf{z}^{1}(\lambda) \oplus \mathbf{z}^{2}(\lambda)$. Then $R$ is l-regularizable and we have reg $\{R[\mathbf{z}(\lambda)]\} \approx$ $\operatorname{reg}\left\{R^{1}\left[\mathbf{z}^{1}(\lambda)\right]\right\} \cup \operatorname{reg}\left\{R^{2}\left[\mathbf{z}^{2}(\lambda)\right]\right\}$.

Proof. Let $\mathbf{v}^{1}$ and $\mathbf{v}^{2}$ be $l$-regularizing vectors and $Q_{l}^{1}\left[\mathbf{y}^{1}(\lambda)\right]$ and $Q_{l}^{2}\left[\mathbf{y}^{2}(\lambda)\right]$ be the corresponding $l$-regularizing polynomials for Riccati operators $R^{1}$ and $R^{2}$. Then we can write

$$
\begin{aligned}
& R^{1}[\mathbf{z}(\lambda)]=R^{1}\left[\mathbf{y}^{1}(\lambda)+\frac{\mathbf{v}^{1}}{\lambda-\xi}\right]=Q_{l}^{1}\left[\mathbf{y}^{1}(\xi)\right] \cdot \mathbf{s}^{1}(\lambda)+\mathbf{r}^{1}(\lambda) \\
& R^{2}[\mathbf{z}(\lambda)]=R^{2}\left[\mathbf{y}^{2}(\lambda)+\frac{\mathbf{v}^{2}}{\lambda-\xi}\right]=Q_{l}^{2}\left[\mathbf{y}^{2}(\xi)\right] \cdot \mathbf{s}^{2}(\lambda)+\mathbf{r}^{2}(\lambda)
\end{aligned}
$$

From these formulas it immediately follows that the Riccati operator $R$ has two $l$ regularizing vectors $\mathbf{v}^{1} \oplus \mathbf{0}$ and $\mathbf{0} \oplus \mathbf{v}^{2}$ and two corresponding $l$-regularizing polynomials $Q_{l}^{1}[\mathbf{y}(\xi)]=Q_{l}^{1}\left[\mathbf{y}^{1}(\xi) \oplus \mathbf{0}\right]$ and $Q_{l}^{2}[\mathbf{y}(\xi)]=Q_{l}^{2}\left[\mathbf{0} \oplus \mathbf{y}^{2}(\xi)\right]$. This completes the proof.

DEFINITION 2.12. If a $l$-regularizable Riccati operator $R$ can be constructed from two or more $l$-regularizable Riccati operators by formula $(2.24)$, we call this operator reducible. Otherwise, we call it irreducible.

Now we are ready to introduce a very important notion of Riccatians.

Definition 2.13. The minimal and irreducible $l$-regularizable Riccati operator we shall call $l$-simple. The particular Riccati polynomials associated with $l$-simple Riccati operators we call Riccatians of genus $l$ or, simply, Riccatians.

Now we see that in order to solve Problem 2.2 it is sufficient to solve the follewin auxiliaty problem:

Problem 2.4. Find all systems of Riccatians.

In next section we start discussion of this problem restricting ourselves by looking only for Riccatians of genus 1 .

3. Construction of Riccatians. This section is devoted to explicit construction of some simplest systems of Riccatians. We start with most general expressions of Riccati operators of a priori given age and, using general prescriptions given in previous section, try to find those forms of these operators for which they become simple. Here we analyze from this point of view three general Riccati operators of ages $|2\rangle,|2,2\rangle$ and $|2,3\rangle$.

3.1. Riccati operators of age $|2\rangle$. The set of Riccati operators of age $|2\rangle$ is defined by the formula

$$
R_{2}[z(\lambda)]=A_{2} \partial z(\lambda)+B_{2} z^{2}(\lambda)
$$

in which $A_{2}$ and $B_{2}$ are arbitrary numerical parameters. In order to find for which values of these parameters the polynomial $R_{2}[z(\lambda)]$ becomes Riccatian, we should analyse its residues and construct corresponding regularizator. 
From general formulas of previous section it follows that the operator (3.1) has two residues

$$
\begin{gathered}
R_{2,0}[y(\xi)]=-A_{2} v+B_{2} v^{2}, \\
R_{2,1}[y(\xi)]=2 B_{2} v y(\xi),
\end{gathered}
$$

and conditions for these residues to have a common divisor of first degree

$$
Q[y(\xi)]=Q \cdot y(\xi)
$$

are

$$
\begin{gathered}
-A_{2} v+B_{2} v^{2}=0, \\
B_{2} v=e_{2} Q .
\end{gathered}
$$

Here $e_{2}$ is some arbitrary constant. We see that for any choice of coefficients $A_{2}$ and $B_{2}$, there exists such value of $e_{2}$ for which system (3.4) has a single non-zero solution for $v$. So that all operators (3.4) are regularizable. Because operators (3.1) are also irreducible and non-minimizible, we can assert that they are simple and polynomials (3.1) are Riccatians for any non-zero values of $A_{2}$ and $B_{2}$.

In order to find the canonical form of these Riccatians, we should take

$$
v=1 .
$$

Substituting (3.5) into (3.4), we find that $A_{2}=B_{2}$. Without loss of generality we can also take $A_{2}=B_{2}=1$ which corresponds to a special choice of normalization. This results in the following final expressions:

$$
R_{2}[z(\lambda)]=\partial z(\lambda)+z^{2}(\lambda)
$$

for the Riccatian and

$$
v=1, \quad Q[y(\xi)]=y(\xi),
$$

for its regularizator.

3.2. Riccati operators of age $|2,2\rangle$. Let us now consider Riccati operators of age $|2,2\rangle$ the most general form of which is given by formulas

$$
\begin{aligned}
& R_{2}[\mathbf{z}(\lambda)]=A_{2}^{i} \partial z_{i}(\lambda)+B_{2}^{i k} z_{i}(\lambda) z_{k}(\lambda), \\
& \bar{R}_{2}[\mathbf{z}(\lambda)]=\bar{A}_{2}^{i} \partial z_{i}(\lambda)+\bar{B}_{2}^{i k} z_{i}(\lambda) z_{k}(\lambda),
\end{aligned}
$$

in which $A_{2}^{i}, B_{2}^{i k}$ and $\bar{A}_{2}^{i}, \bar{B}_{2}^{i k}$, are some arbitrary parameters. The indices $i$ and $k$ in (3.8) take the values 1 and 2 and the summation over repeated indices is assumed.

As before, in order to find the values of parameters for which the polynomials (3.8a) and $(3.8 \mathrm{~b})$ become Riccatians, we should first look at the residues of these polynomials

$$
\begin{gathered}
R_{2,0}[\mathbf{y}(\xi)]=-A_{2}^{i} v_{i}+B_{2}^{i k} v_{i} v_{k}, \\
R_{2,1}[\mathbf{y}(\xi)]=2 B_{2}^{i k} v_{i} y_{k}(\xi),
\end{gathered}
$$

and

$$
\begin{gathered}
\bar{R}_{2,0}[\mathbf{y}(\xi)]=-\bar{A}_{2}^{i} v_{i}+\bar{B}_{2}^{i k} v_{i} v_{k}, \\
\bar{R}_{2,1}[\mathbf{y}(\xi)]=2 \bar{B}_{2}^{i k} v_{i} y_{k}(\xi) .
\end{gathered}
$$


The conditions for all these residues to have a common divisor of degree one,

$$
Q[y(\xi)]=Q^{l} y_{l}(\xi),
$$

are, respectively,

$$
\begin{gathered}
-A_{2}^{i} v_{i}+B_{2}^{i k} v_{i} v_{k}=0, \\
B_{2}^{l i} v_{i}=e_{2} Q^{l}
\end{gathered}
$$

and

$$
\begin{gathered}
-\bar{A}_{2}^{i} v_{i}+\bar{B}_{2}^{i k} v_{i} v_{k}=0, \\
\bar{B}_{2}^{l i} v_{i}=\bar{e}_{2} Q^{l},
\end{gathered}
$$

where $e_{2}$ and $\bar{e}_{2}$ are some non-zero numbers.

Comparing formulas $(3.12 \mathrm{~b})$ and $(3.13 \mathrm{~b})$, we can write

$$
\frac{1}{e_{2}} B_{2}^{l i} v_{i}=\frac{1}{\bar{e}_{2}} \bar{B}_{2}^{l i} v_{i}
$$

or, in matrix notations,

$$
\begin{aligned}
& B_{2} \bar{B}_{2}^{-1} \mathbf{v}=\epsilon_{2} \mathbf{v}, \\
& \bar{B}_{2} B_{2}^{-1} \mathbf{v}=\bar{\epsilon}_{2} \mathbf{v},
\end{aligned}
$$

where $\epsilon=e_{2} \bar{e}_{2}^{-1}$ and $\bar{\epsilon}=\bar{e}_{2} e_{2}^{-1}$. Relations (3.15) mean that vectors $\mathbf{v}$ are the eigenvectors of the matrices $B_{2} \bar{B}_{2}^{-1}$ and $\bar{B}_{2} B_{2}^{-1}$, while the numbers $\epsilon_{2}$ and $\bar{\epsilon}_{2}$ are their eigenvalues. Because $B_{2} \bar{B}_{2}^{-1}$ and $\bar{B}_{2} B_{2}^{-1}$ are $2 \times 2$ matrices, the equations (3.15) should have at least two linearly independent solutions which we denote by $\mathbf{v}^{n}=\left\{v_{i}^{n}\right\}$. The linear independence of these vectors enables one to choose such a basis in two-dimensional vector space, in which they become orthonormal repers

$$
v_{i}^{n}=\delta_{i}^{n} .
$$

Denoting the corresponding eigenvalues by $\epsilon_{2}^{n}$ and $\bar{\epsilon}_{2}^{n}$ and substituting (3.16) into (3.15), we obtain

$$
\begin{aligned}
& B_{2}^{l n}=\epsilon^{n} \bar{B}_{2}^{l n}, \\
& \bar{B}_{2}^{l n}=\bar{\epsilon}^{n} B_{2}^{l n} .
\end{aligned}
$$

Let us now remember that both matrices $B_{2}^{l n}$ and $\bar{B}_{2}^{l n}$ are symmetric. Permuting the indices $l$ and $m$ in (3.17) and subtracting obtained relations from each other we obtain

$$
\begin{aligned}
& \left(\epsilon^{n}-\epsilon^{l}\right) \bar{B}_{2}^{l n}=0, \\
& \left(\bar{\epsilon}^{n}-\bar{\epsilon}^{l}\right) B_{2}^{l n}=0 .
\end{aligned}
$$

These relations can be satisfied in two cases: 1) if $\epsilon^{1}=\epsilon^{2}=\varepsilon\left(\right.$ or $\bar{\epsilon}^{1}=\bar{\epsilon}^{2}=\varepsilon$ ) and $B_{2}^{\text {ln }}$ (or $\bar{B}_{2}^{l n}$ ) is arbitrary, and 2) if $\epsilon^{1} \neq \epsilon^{2}\left(\right.$ or $\left.\bar{\epsilon}^{1} \neq \bar{\epsilon}^{2}\right)$ and $B_{2}^{12}=0$ (or $\left.\bar{B}_{2}^{12}=0\right)$.

Consider the first case. According to (3.17), the matrices $B_{2}^{l n}$ and $\bar{B}_{2}^{l n}$ are proportional to each other. Substitution of (3.16) into (3.12a) and (3.13a) demonstrates the proportionality of vectors $A_{2}^{l}$ and $\bar{A}_{2}^{l}$. This means that the polynomials $R_{2}[\mathbf{z}(\lambda)]$ and $\bar{R}_{2}[\mathbf{z}(\lambda)]$, should also be proportional to each other, and thus, cannot be interpreted as Riccatians.

Let us now consider the second case. In this case the matrices $B_{2}^{l n}$ and $\bar{B}_{2}^{l n}$ are diagonal. Denoting their diagonal elements by $B_{2}^{l}$ and $\bar{B}_{2}^{l}$ and substituting (3.16) into 
(3.12a) and (3.13a), we find that $A_{2}^{l}=B_{2}^{l}$ and $\bar{A}_{2}^{l}=\bar{B}_{2}^{l}$. This gives

$$
\begin{aligned}
& R_{2}[\mathbf{z}(\lambda)]=A_{2}^{1}\left\{\partial z_{1}(\lambda)+2 z_{1}^{2}(\lambda)\right\}+A_{2}^{2}\left\{\partial z_{2}(\lambda)+2 z_{2}^{2}(\lambda)\right\} \\
& \bar{R}_{2}[\mathbf{z}(\lambda)]=\bar{A}_{2}^{1}\left\{\partial z_{1}(\lambda)+2 z_{1}^{2}(\lambda)\right\}+\bar{A}_{2}^{2}\left\{\partial z_{2}(\lambda)+2 z_{2}^{2}(\lambda)\right\}
\end{aligned}
$$

We see that the Riccati operator defined by relations (3.19) is reducible, and therefore, the polynomials $R_{2}[\mathbf{z}(\lambda)]$ and $\bar{R}_{2}[\mathbf{z}(\lambda)]$ cannot be considered as Riccatians.

Summarizing, we can assert that there are no simple Riccati operators of age $|2,2\rangle$.

3.3. Riccati operators of age $|2,3\rangle$. Consider a general Riccati operator of age $|2,3\rangle$ determined by formulas

$$
\begin{gathered}
R_{2}[z(\lambda)]=A_{2}^{i} \partial z_{i}(\lambda)+B_{2}^{i k} z_{i}(\lambda) z_{k}(\lambda), \\
R_{3}[z(\lambda)]=A_{3}^{i} \partial^{2} z_{i}(\lambda)+B_{3}^{i, k} \partial z_{i}(\lambda) z_{k}(\lambda)+C_{3}^{i k l} z_{i}(\lambda) z_{k}(\lambda) z_{l}(\lambda),
\end{gathered}
$$

and try to find conditions for its coefficients under which the polynomials $R_{2}[z(\lambda)]$ and $R_{3}[z(\lambda)]$ become Riccatians. As before, the indices $i, k$ and $l$ in (3.20) take the values 1 and 2 , and the summation over repeated indices is assumed.

The residues of polynomials (3.20a) and (3.20b) have the form

$$
\begin{gathered}
R_{2,0}[y(\xi)]=-A_{2}^{i} v_{i}+B_{2}^{i k} v_{i} v_{k}, \\
R_{2,1}[y(\xi)]=2 B_{2}^{i k} v_{i} y_{k}(\xi),
\end{gathered}
$$

and

$$
\begin{gathered}
R_{3,0}[y(\xi)]=2 A_{3}^{i} v_{i}-B_{3}^{i, k} v_{i} v_{k}+C_{3}^{i k l} v_{i} v_{k} v_{l}, \\
R_{3,1}[y(\xi)]=\left(-B_{3}^{i, l} v_{i}+3 C_{3}^{i k l} v_{i} v_{k}\right) y_{l}(\xi), \\
R_{3,2}[y(\xi)]=\left(B^{l, i} v_{i}-B_{3}^{i, l} v_{i}+3 C_{3}^{i k l} v_{i} v_{k}\right) \dot{y}_{l}(\xi)+3 C_{3}^{i k l} v_{i} y_{k}(\xi) y_{l}(\xi) .
\end{gathered}
$$

The conditions for all these residues to have a common divisor of first degree

$$
Q[y(\xi)]=Q^{l} y_{l}(\xi)
$$

are

$$
\begin{gathered}
-A_{2}^{i} v_{i}+B_{2}^{i k} v_{i} v_{k}=0, \\
B_{2}^{l i} v_{i}=e_{2} Q^{l},
\end{gathered}
$$

for the first polynomial, and

$$
\begin{gathered}
2 A_{3}^{i} v_{i}-B_{3}^{i, k} v_{i} v_{k}+C_{3}^{i k l} v_{i} v_{k} v_{l}=0 \\
B_{3}^{i, l} v_{i}-3 C_{3}^{i k l} v_{i} v_{k}=e_{3} Q^{l} \\
\left(B_{3}^{l, i}-B_{3}^{i, l}\right) v_{i}+3 C_{3}^{i k l} v_{i} v_{k}=0 \\
3 C_{3}^{i k l} v_{i}=f_{3}^{k} Q^{l}+f_{3}^{l} Q^{k}
\end{gathered}
$$

for the second polynomial. Here $e_{2}$ and $e_{3}$ are some unknown numbers and $f_{3}^{i}$ is some unknown vector. Below we shall assume that all these quantities differ from zero.

We start with the second system. Multiplying (3.25c) by $v_{l}$ we obtain the condition

$$
\left(B_{3}^{l, i}-B_{3}^{i, l}\right) v_{i} v_{l}+3 C_{3}^{i k l} v_{i} v_{k} v_{l}=0,
$$

the comparizon of which with (3.25a) gives

$$
-A_{3}^{i} v_{i}+B_{3}^{i k} v_{i} v_{k}=0 .
$$


This equation is similar to equation (3.24a) from the first system. Comparing (3.25b) with $(3.25 \mathrm{c})$ we get another equation

$$
B_{3}^{l, i} v_{i}=e_{3} Q^{l}
$$

which is obviously similar to equation (3.24b). From (3.24b) and (3.28) it follows that

$$
\frac{1}{e_{2}} B_{2}^{l i} v_{i}=\frac{1}{e_{3}} B_{3}^{l, i} v_{i}
$$

or, equivalently, in the matrix form,

$$
B_{3} B_{2}^{-1} \mathbf{v}=\epsilon \mathbf{v} .
$$

The last condition means that the vectors $\mathbf{v}$ are the eigenvectors of the matrix $B_{3} B_{2}^{-1}$, while the fractions $\epsilon=e_{3} e_{2}^{-1}$ are its eigenvalues.

The next step is to find the vectors $f_{3}^{i}$. Multiplying (3.25d) by $v_{k} v_{l}$ and using (3.26) we obtain

$$
\left(f_{3}^{k} v_{k}\right)\left(Q^{l} v_{l}\right)=0
$$

which gives

$$
\left(f_{3}^{k} v_{k}\right)=0
$$

provided that the second factor in (3.31) differs from zero. Now multiplying (3.25d) by $v_{k}$ and using (3.32) we get the condition

$$
3 C_{3}^{i k l} v_{i} v_{k}=f_{3}^{l}\left(Q^{k} v_{k}\right)
$$

which together with another condition

$$
B_{3}^{i, k} v_{i} v_{k}=e_{3}\left(Q^{k} v_{k}\right),
$$

obtained by multiplying (3.28) by $v_{k}$, enables one to determine $f_{3}^{l}$ :

$$
f_{3}^{l}=e_{3} \frac{3 C_{3}^{i k l} v_{i} v_{k}}{B_{3}^{i, k} v_{i} v_{k}} .
$$

After using (3.25) we get

$$
f_{3}^{l}=e_{3} \frac{\left(B_{3}^{l, i}-B_{3}^{i, l}\right) v_{i}}{B_{3}^{i, k} v_{i} v_{k}} .
$$

Substitution of (3.36) into (3.25d) gives

$$
3 C_{3}^{i k l} v_{i}=\left(e_{3} Q^{k}\right) \frac{\left(B_{3}^{l, i}-B_{3}^{i, l}\right) v_{i}}{B_{3}^{i, j} v_{i} v_{j}}+\left(e_{3} Q^{l}\right) \frac{\left(B_{3}^{k, i}-B_{3}^{i, k}\right) v_{i}}{B_{3}^{i, j} v_{i} v_{j}},
$$

or, after taking into account (3.28),

$$
3 C_{3}^{i k l} v_{i}=\frac{\left(B_{3}^{i, k} B_{3}^{l, j}+B_{3}^{i, l} B_{3}^{k, j}-2 B_{3}^{k, i} B_{3}^{l, j}\right) v_{i} v_{j}}{B_{3}^{i, j} v_{i} v_{j}} .
$$

This formula will play the central role in our further considerations.

We know that $B_{3}$ and $B_{2}$ are $2 \times 2$ matrices, and therefore the spectral problem (3.30) should have two linearly independent eigenvectors. Denote these eigenvectors by 
$\mathbf{v}^{n}=\left\{v_{i}^{n}\right\}$ and note that the basis in the space to which they belong, can always be chosen in such a way as to guarantee their coincidence with orthonormal repers

$$
v_{i}^{n}=\delta_{i}^{n} .
$$

Substituting (3.39) into (3.38) we then obtain

$$
3 C_{3}^{n k l}=\frac{B_{3}^{n, k} B_{3}^{l, n}+B_{3}^{n, l} B_{3}^{k, n}-2 B_{3}^{k, n} B_{3}^{l, n}}{B_{3}^{n, n}} .
$$

Let $\epsilon^{n}$ denote the eigenvalues of the matrix $B_{3} B_{2}^{-1}$ corresponding to the orthonormalized eigenvectors $\mathbf{v}^{n}$. Then from (3.39) and (3.29) it follows that

$$
B_{3}^{l, n}=\epsilon^{n} B_{2}^{l n} \text {. }
$$

Substituting (3.41) into (3.40) and taking into account the fact that the matrix $B_{2}$ is symmetric, we obtain

$$
3 C_{3}^{n k l}=\left(\epsilon^{n}+\epsilon^{l}-2 \epsilon^{k}\right) \frac{B_{2}^{k n} B_{2}^{k l}}{B_{2}^{k k}} .
$$

Remember now that the tensor $3 C_{3}^{n k l}$ is totally symmetric. At the same time, the expression in the right hand side of (3.42) is automatically symmetric only with respect to indices $n$ and $l$. In order to guarantee the total symmetry of this expression it is sufficient to require its symmetry with respect to indices $l$ and $k$. This gives the condition

$$
\left(\epsilon^{n}+\epsilon^{l}-2 \epsilon^{k}\right) \frac{B_{2}^{k n}}{B_{2}^{k k}} B_{2}^{k l}=\left(\epsilon^{n}+\epsilon^{k}-2 \epsilon^{l}\right) \frac{B_{2}^{l n}}{B_{2}^{l l}} B_{2}^{k l} .
$$

Taking $k=n$ in (3.43) we obtain

$$
\left(\epsilon^{l}-\epsilon^{n}\right) B^{l n}\left(1+2 \frac{B_{2}^{l n}}{B_{2}^{l l}}\right)=0 .
$$

Now note that the relation (3.44) can be realized in three cases: 1) if $\epsilon^{1}=\epsilon^{2}$ and $B_{2}^{l n}$ is arbitrary, 2) if $\epsilon^{1} \neq \epsilon^{2}$ and $B_{2}^{l n}$ is diagonal, and 3) if $\epsilon^{1} \neq \epsilon^{2}$ and $\left(1+2 B_{2}^{l n} / B_{2}^{l l}\right)=0$. Repeating the reasonings of the previous subsection it is not difficult to show that the first possibility leads to a system of dependent Riccati polynomials which cannot be interpreted as Riccatians, and the second possibility leads to a reducible system of Riccati polynomials which also cannot be interpreted as Riccatians.

Consider the third case. In this case we have

$$
B_{2}^{l n}=-\frac{1}{2} B_{2}^{l l} .
$$

Because of the symmetry of matrix $B_{2}$ we also have

$$
B_{2}^{l n}=-\frac{1}{2} B_{2}^{n n},
$$

which means that

$$
B_{2}^{11}=B_{2}^{22}=B, \quad B_{2}^{12}=B_{2}^{21}=-\frac{B}{2} .
$$

Substituting (3.39) into (3.24a) we find

$$
A_{2}^{1}=B, \quad A_{2}^{2}=B .
$$


In order to compute the matrix $B_{3}^{i, k}$ by means of formula (3.41) we need the eigenvalues $e^{1}$ and $e^{2}$. However, it is easily seen that there are no formulas imposing some constraints on $e^{1}$ and $e^{2}$. This means that these eigenvalues can be considered as free parameters. For the sake of further convenience, we introduce instead of $e^{1}$ and $e^{2}$ two other parameters, $C$ and $D$, by formulas

$$
e^{1}=2 \frac{C+D}{B} \quad e^{2}=2 \frac{C-D}{B} .
$$

Then, using (3.49), (3.41) and (3.47) we get

$$
B_{3}^{1,1}=2 C+2 D, \quad B_{3}^{2,2}=2 C-2 D, \quad B_{3}^{1,2}=-C+D, \quad B_{3}^{2,1}=-C-D .
$$

Formula (3.27) after applying to it (3.39) gives

$$
A_{3}^{1}=C+D, \quad A_{3}^{2}=C-D
$$

Finally, using (3.42) we obtain:

$$
\begin{gathered}
C_{3}^{111}=0, \quad C_{3}^{222}=0 \\
C_{3}^{112}=C_{3}^{121}=C_{3}^{211}=2 D \\
C_{3}^{221}=C_{3}^{212}=C_{3}^{122}=-2 D .
\end{gathered}
$$

Collecting the obtained expressions for the coefficients of Riccati polynomials and writing down final and most general expressions for Riccatians (3.20a) and (3.20b), we can see that the polynomial proportional to $C$ is nothing else than the derivative of $R_{2}[z(\lambda)]$. Thus, without loss of generality, we can take $C=0$. Choosing the remaining normalization coefficients as $B=1$ and $D=1$, we obtain final expression for the system of two Riccatians of orders 2 and 3 .

$$
\begin{gathered}
R_{2}[z(\lambda)]=\partial z_{1}(\lambda)+\partial z_{2}(\lambda)+z_{1}(\lambda) z_{2}(\lambda)+z_{2}(\lambda) z_{2}(\lambda)-z_{1}(\lambda) z_{2}(\lambda) \\
R_{3}[z(\lambda)]=\partial^{2} z_{1}(\lambda)-\partial^{2} z_{2}(\lambda)+2 \partial z_{1}(\lambda) z_{1}(\lambda)-2 \partial z_{2}(\lambda) z_{2}(\lambda) \\
+\partial z_{1}(\lambda) z_{2}(\lambda)-\partial z_{2}(\lambda) z_{1}(\lambda)++2 z_{1}(\lambda) z_{2}(\lambda) z_{2}(\lambda)-2 z_{2}(\lambda) z_{2}(\lambda) z_{1}(\lambda) .
\end{gathered}
$$

The regularizator of the system (3.53) consists of two elements

$$
\begin{aligned}
& v^{1}=(1,0), \quad Q^{1}[z(\lambda)]=z_{1}(\lambda)-\frac{1}{2} z_{2}(\lambda), \\
& v^{2}=(0,1), \quad Q^{2}[z(\lambda)]=z_{2}(\lambda)-\frac{1}{2} z_{1}(\lambda) .
\end{aligned}
$$

These expressions immediately follow from formulas (3.24a) and (3.28) after taking into account explicit expressions for matrices $B_{2}, B_{3}$ and numbers $e_{2}, e_{3}$.

4. Some other examples of Riccatians. The calculations given in previous section can be repeated for other Riccati operators. Being essentially the same they, however, become technically more and more complicated with increasing the age of Riccati operators. For this reason we were forced to use the programs of analytic calculations like REDUCE and MATHEMATICA. We have analysed all Riccati operators of ages $\left|n_{1}, \ldots, n_{r}\right\rangle$ with $r \leq 3$ and $n_{r} \leq 6$. We present here the results of such calculations ommiting details of their derivations. Below we give only the list of simple Riccati operators generating the 
systems of Riccatians. For the sake of completeness this list will also contain the cases already discussed in previous section.

4.1. Riccati operator of age $|2\rangle$.

Riccatian:

$$
R_{2}[\mathbf{z}]=\partial z_{1}+z_{1}^{2}
$$

Regularizator:

$$
\mathbf{v}^{1}=1, \quad Q^{1}[\mathbf{y}]=y .
$$

4.2. Riccati operator of age $|2,3\rangle$. Riccatians $\left({ }^{4}\right)$ :

$$
\begin{gathered}
R_{2}[\mathbf{z}]=\partial z_{1}+\partial z_{2}+z_{1}^{2}-z_{1} z_{2}+z_{2}^{2}, \\
R_{3}[\mathbf{z}]=\partial^{2} z_{1}+2 \partial z_{1} z_{1}-\partial z_{2} z_{1}+z_{1}^{2} z_{2}-z_{1} z_{2}^{2} .
\end{gathered}
$$

Regularizator:

$$
\begin{aligned}
& \mathbf{v}^{1}=(1,0), \quad Q^{1}[\mathbf{y}]=y_{1}-\frac{1}{2} y_{2}, \\
& \mathbf{v}^{2}=(0,1), \quad Q^{2}[\mathbf{y}]=y_{2}-\frac{1}{2} y_{1} .
\end{aligned}
$$

4.3. Riccati operator of age $|2,4\rangle$.

Riccatians:

$$
\begin{gathered}
R_{2}[\mathbf{z}]=2 \partial z_{1}+\partial z_{2}+2 z_{1}^{2}-2 z_{1} z_{2}+z_{2}^{2} \\
R_{4}[\mathbf{z}]=\partial^{3} z_{1}+2 \partial^{2} z_{1} z_{1}-\partial^{2} z_{2} z_{1}+\left(\partial z_{1}\right)^{2}-\partial z_{1} \partial z_{2} \\
-2 \partial z_{1} z_{1}^{2}+4 \partial z_{1} z_{1} z_{2}-\partial z_{1} z_{2}^{2}+\partial z_{2} z_{1}^{2}-2 \partial z_{2} z_{1} z_{2}-z_{1}^{4}+2 z_{1}^{3} z_{2}-z_{1}^{2} z_{2}^{2} .
\end{gathered}
$$

Regularizator:

$$
\begin{array}{ll}
\mathbf{v}^{1}=(1,0), & Q^{1}[\mathbf{y}]=2 y_{1}-y_{2}, \\
\mathbf{v}^{2}=(0,1), & Q^{2}[\mathbf{y}]=y_{2}-y_{1} .
\end{array}
$$

4.4. Riccati operator of age $|2,6\rangle$.

Riccatians:

$$
\begin{gathered}
R_{2}[\mathbf{z}]=3 \partial z_{1}+\partial z_{2}+3 z_{1}^{2}-3 z_{1} z_{2}+z_{2}^{2} \\
R_{6}[\mathbf{z}]=5 \partial^{5} z_{1}+\partial^{5} z_{2}+10 \partial^{4} z_{1} z_{1}-3 \partial^{4} z_{1} z_{2}-5 \partial^{4} z_{2} z_{1}+2 \partial^{4} z_{2} z_{2} \\
+17 \partial^{3} z_{1} \partial z_{1}-19 \partial^{3} z_{1} \partial z_{2}-21 \partial^{3} z_{2} \partial z_{1}+7 \partial^{3} z_{2} \partial z_{2} \\
+10\left(\partial^{2} z_{1}\right)^{2}-30 \partial^{2} z_{1} \partial^{2} z_{2}+5\left(\partial^{2} z_{2}\right)^{2}-23 \partial^{3} z_{1} z_{1}^{2} \\
+27 \partial^{3} z_{1} z_{1} z_{2}-7 \partial^{3} z_{1} z_{2}^{2}-\partial^{3} z_{2} z_{1}^{2}-\partial^{3} z_{2} z_{1} z_{2} \\
-\partial^{3} z_{2} z_{2}^{2}-126 \partial^{2} z_{1} \partial z_{1} z_{1}+63 \partial^{2} z_{1} \partial z_{1} z_{2}+42 \partial^{2} z_{1} \partial z_{2} z_{1} \\
-21 \partial^{2} z_{1} \partial z_{2} z_{2}+23 \partial^{2} z_{2} \partial z_{1} z_{1}-12 \partial^{2} z_{2} \partial z_{1} z_{2}-\partial^{2} z_{2} \partial z_{2} z_{1} \\
-6 \partial^{2} z_{2} \partial z_{2} z_{2}-42\left(\partial z_{1}\right)^{3}+46\left(\partial z_{1}\right)^{2} \partial z_{2}-0 \partial z_{1}\left(\partial z_{2}\right)^{2} \\
-2\left(\partial z_{2}\right)^{3}-46 \partial^{2} z_{1} z_{1}^{3}+57 \partial^{2} z_{1} z_{1}^{2} z_{2}-21 \partial^{2} z_{1} z_{1} z_{2}^{2} \\
+3 \partial^{2} z_{1} z_{2}^{3}+23 \partial^{2} z_{2} z_{1}^{3}-29 \partial^{2} z_{2} z_{1}^{2} z_{2}+11 \partial^{2} z_{2} z_{1} z_{2}^{2}
\end{gathered}
$$

$\left({ }^{4}\right)$ Here the role of Riccatian $R_{3}$ plays the combination $R_{3}+\partial R_{2}$ of Riccatians (3.53). 


$$
\begin{gathered}
-2 \partial^{2} z_{2} z_{2}^{3}-114\left(\partial z_{1}\right)^{2} z_{1}^{2}+90\left(\partial z_{1}\right)^{2} z_{1} z_{2}-17\left(\partial z_{1}\right)^{2} z_{2}^{2} \\
+122 \partial z_{1} \partial z_{2} z_{1}^{2}-98 \partial z_{1} \partial z_{2} z_{1} z_{2}+22 \partial z_{1} \partial z_{2} z_{2}^{2}-28\left(\partial z_{2}\right)^{2} z_{1}^{2} \\
+22\left(\partial z_{2}\right)^{2} z_{1} z_{2}-6\left(\partial z_{2}\right)^{2} z_{2}^{2}+12 \partial z_{1} z_{1}^{4}-48 \partial z_{1} z_{1}^{3} z_{2} \\
+50 \partial z_{1} z_{1}^{2} z_{2}^{2}-18 \partial z_{1} z_{1} z_{2}^{3}+2 \partial z_{1} z_{2}^{4}-2 \partial z_{2} z_{1}^{4} \\
+16 \partial z_{2} z_{1}^{3} z_{2}-16 \partial z_{2} z_{1}^{2} z_{2}^{2}+4 \partial z_{2} z_{1} z_{2}^{3}+4 z_{1}^{6} \\
-12 z_{1}^{5} z_{2}+13 z_{1}^{4} z_{2}^{2}-6 z_{1}^{3} z_{2}^{3}+z_{1}^{2} z_{2}^{4} .
\end{gathered}
$$

Regularizator:

$$
\begin{array}{cl}
\mathbf{v}^{1}=(1,0), & Q^{1}[\mathbf{y}]=3 y_{1}-\frac{3}{2} y_{2}, \\
\mathbf{v}^{2}=(0,1), & Q^{2}[\mathbf{y}]=y_{2}-\frac{3}{2} y_{1} .
\end{array}
$$

4.5. Riccati operators of age $|2,3,4\rangle$.

Riccatians:

$$
\begin{gathered}
R_{2}[\mathbf{z}]=\partial z_{1}+\partial z_{2}+\partial z_{3}+z_{1}^{2}-z_{1} z_{2}+z_{2}^{2}-z_{2} z_{3}+z_{3}^{2} \\
R_{3}[\mathbf{z}]=2 \partial^{2} z_{1}+\partial^{2} z_{2} \\
+4 \partial z_{1} z_{1}-\partial z_{1} z_{2}-2 \partial z_{2} z_{1}+2 \partial z_{2} z_{2}-\partial z_{3} z_{2}+z_{1}^{2} z_{2}-z_{1} z_{2}^{2}+z_{2}^{2} z_{3}-z_{2} z_{3}^{2}, \\
R_{4}[\mathbf{z}]=\partial^{3} z_{1}+2 \partial^{2} z_{1} z_{1}-\partial^{2} z_{2} z_{1}+2\left(\partial z_{1}\right)^{2}-\partial z_{1} \partial z_{2} \\
-\partial z_{1} \partial z_{3}+2 \partial z_{1} z_{1} z_{2}-\partial z_{1} z_{2}^{2}+\partial z_{1} z_{2} z_{3}-\partial z_{1} z_{3}^{2} \\
+\partial z_{2} z_{1}^{2}-2 \partial z_{2} z_{1} z_{2}-\partial z_{3} z_{1}^{2}+\partial z_{3} z_{1} z_{2}+z_{1}^{2} z_{2} z_{3} \\
-z_{1}^{2} z_{3}^{2}-z_{1} z_{2}^{2} z_{3}+z_{1} z_{2} z_{3}^{2}
\end{gathered}
$$

Regularizator:

$$
\begin{gathered}
\mathbf{v}^{1}=(1,0,0), \quad Q^{1}[\mathbf{y}]=y_{1}-\frac{1}{2} y_{2}, \\
\mathbf{v}^{2}=(0,1,0), \quad Q^{2}[\mathbf{y}]=y_{2}-\frac{1}{2} y_{1}-\frac{1}{2} y_{3}, \\
\mathbf{v}^{3}=(0,0,1), \quad Q^{3}[\mathbf{y}]=y_{3}-\frac{1}{2} y_{2} .
\end{gathered}
$$

4.6. Riccati operator of age $|2,4,6\rangle$. First case.

Riccatians:

$$
\begin{gathered}
R_{2}[\mathbf{z}]=2 \partial z_{1}+2 \partial z_{2}+\partial z_{3}+2 z_{1}^{2}-2 z_{1} z_{2}+2 z_{2}^{2}-2 z_{2} z_{3}+z_{3}^{2}, \\
R_{4}[\mathbf{z}]=6 \partial^{3} z_{1}+3 \partial^{3} z_{2}+\partial^{3} z_{3}+12 \partial^{2} z_{1} z_{1}-3 \partial^{2} z_{1} z_{2} \\
-6 \partial^{2} z_{2} z_{1}+6 \partial^{2} z_{2} z_{2}-2 \partial^{2} z_{2} z_{3}-3 \partial^{2} z_{3} z_{2}+2 \partial^{2} z_{3} z_{3} \\
+11\left(\partial z_{1}\right)^{2}-11 \partial z_{1} \partial z_{2}-2 \partial z_{1} \partial z_{3}+5\left(\partial z_{2}\right)^{2}-5 \partial z_{2} \partial z_{3} \\
+2\left(\partial z_{3}\right)^{2}-2 \partial z_{1} z_{1}^{2}+8 \partial z_{1} z_{1} z_{2}-5 \partial z_{1} z_{2}^{2}+4 \partial z_{1} z_{2} z_{3} \\
-2 \partial z_{1} z_{3}^{2}+\partial z_{2} z_{1}^{2}-4 \partial z_{2} z_{1} z_{2}-2 \partial z_{2} z_{2}^{2}+4 \partial z_{2} z_{2} z_{3} \\
-\partial z_{2} z_{3}^{2}-2 \partial z_{3} z_{1}^{2}+2 \partial z_{3} z_{1} z_{2}+\partial z_{3} z_{2}^{2}-2 \partial z_{3} z_{2} z_{3} \\
-z_{1}^{4}+2 z_{1}^{3} z_{2}-3 z_{1}^{2} z_{2}^{2}+4 z_{1}^{2} z_{2} z_{3}-2 z_{1}^{2} z_{3}^{2}+2 z_{1} z_{2}^{3} \\
-4 z_{1} z_{2}^{2} z_{3}+2 z_{1} z_{2} z_{3}^{2}-z_{2}^{4}+2 z_{2}^{3} z_{3}-z_{2}^{2} z_{3}^{2},
\end{gathered}
$$


(4.11c)

$$
\begin{aligned}
& R_{6}[\mathbf{z}]=\partial^{5} z_{1}+2 \partial^{4} z_{1} z_{1}-\partial^{4} z_{2} z_{1}+7 \partial^{3} z_{1} \partial z_{1}-2 \partial^{3} z_{1} \partial z_{2} \\
& -\partial^{3} z_{1} \partial z_{3}-3 \partial^{3} z_{2} \partial z_{1}-\partial^{3} z_{3} \partial z_{1}+6\left(\partial^{2} z_{1}\right)^{2}-4 \partial^{2} z_{1} \partial^{2} z_{2} \\
& -2 \partial^{2} z_{1} \partial^{2} z_{3}-\partial^{3} z_{1} z_{1}^{2}+3 \partial^{3} z_{1} z_{1} z_{2}-2 \partial^{3} z_{1} z_{2}^{2}+2 \partial^{3} z_{1} z_{2} z_{3} \\
& -\partial^{3} z_{1} z_{3}^{2}+\partial^{3} z_{2} z_{1}^{2}-2 \partial^{3} z_{2} z_{1} z_{2}-\partial^{3} z_{3} z_{1}^{2}+\partial^{3} z_{3} z_{1} z_{2} \\
& -2 \partial^{2} z_{1} \partial z_{1} z_{1}+7 \partial^{2} z_{1} \partial z_{1} z_{2}+4 \partial^{2} z_{1} \partial z_{2} z_{1}-8 \partial^{2} z_{1} \partial z_{2} z_{2} \\
& +4 \partial^{2} z_{1} \partial z_{2} z_{3}-2 \partial^{2} z_{1} \partial z_{3} z_{1}+4 \partial^{2} z_{1} \partial z_{3} z_{2}-4 \partial^{2} z_{1} \partial z_{3} z_{3} \\
& +5 \partial^{2} z_{2} \partial z_{1} z_{1}-6 \partial^{2} z_{2} \partial z_{1} z_{2}+2 \partial^{2} z_{2} \partial z_{1} z_{3}-4 \partial^{2} z_{2} \partial z_{2} z_{1} \\
& +\partial^{2} z_{2} \partial z_{3} z_{1}-4 \partial^{2} z_{3} \partial z_{1} z_{1}+3 \partial^{2} z_{3} \partial z_{1} z_{2}-2 \partial^{2} z_{3} \partial z_{1} z_{3} \\
& +2 \partial^{2} z_{3} \partial z_{2} z_{1}-2\left(\partial z_{1}\right)^{3}+5\left(\partial z_{1}\right)^{2} \partial z_{2}-\left(\partial z_{1}\right)^{2} \partial z_{3} \\
& -5 \partial z_{1}\left(\partial z_{2}\right)^{2}+5 \partial z_{1} \partial z_{2} \partial z_{3}-2 \partial z_{1}\left(\partial z_{3}\right)^{2}-2 \partial^{2} z_{1} z_{1}^{3} \\
& +3 \partial^{2} z_{1} z_{1}^{2} z_{2}-3 \partial^{2} z_{1} z_{1} z_{2}^{2}+4 \partial^{2} z_{1} z_{1} z_{2} z_{3}-2 \partial^{2} z_{1} z_{1} z_{3}^{2} \\
& +\partial^{2} z_{2} z_{1}^{3}-2 \partial^{2} z_{2} z_{1}^{2} z_{2}+2 \partial^{2} z_{2} z_{1}^{2} z_{3}+2 \partial^{2} z_{2} z_{1} z_{2}^{2} \\
& -4 \partial^{2} z_{2} z_{1} z_{2} z_{3}+\partial^{2} z_{2} z_{1} z_{3}^{2}+\partial^{2} z_{3} z_{1}^{2} z_{2}-2 \partial^{2} z_{3} z_{1}^{2} z_{3} \\
& -\partial^{2} z_{3} z_{1} z_{2}^{2}+2 \partial^{2} z_{3} z_{1} z_{2} z_{3}-2\left(\partial z_{1}\right)^{2} z_{1}^{2}+2\left(\partial z_{1}\right)^{2} z_{1} z_{2} \\
& -\left(\partial z_{1}\right)^{2} z_{2}^{2}+2\left(\partial z_{1}\right)^{2} z_{2} z_{3}-\left(\partial z_{1}\right)^{2} z_{3}^{2}-6 \partial z_{1} \partial z_{2} z_{1} z_{2} \\
& +8 \partial z_{1} \partial z_{2} z_{1} z_{3}+2 \partial z_{1} \partial z_{2} z_{2}^{2}-4 \partial z_{1} \partial z_{2} z_{2} z_{3}+\partial z_{1} \partial z_{2} z_{3}^{2} \\
& +2 \partial z_{1} \partial z_{3} z_{1}^{2}+4 \partial z_{1} \partial z_{3} z_{1} z_{2}-8 \partial z_{1} \partial z_{3} z_{1} z_{3}-\partial z_{1} \partial z_{3} z_{2}^{2} \\
& +2 \partial z_{1} \partial z_{3} z_{2} z_{3}-\left(\partial z_{2}\right)^{2} z_{1}^{2}+4\left(\partial z_{2}\right)^{2} z_{1} z_{2}-4\left(\partial z_{2}\right)^{2} z_{1} z_{3} \\
& +3 \partial z_{2} \partial z_{3} z_{1}^{2}-6 \partial z_{2} \partial z_{3} z_{1} z_{2}+4 \partial z_{2} \partial z_{3} z_{1} z_{3}-2\left(\partial z_{3}\right)^{2} z_{1}^{2} \\
& +2\left(\partial z_{3}\right)^{2} z_{1} z_{2}+2 \partial z_{1} z_{1}^{2} z_{2}^{2}-4 \partial z_{1} z_{1}^{2} z_{2} z_{3}+2 \partial z_{1} z_{1}^{2} z_{3}^{2} \\
& -4 \partial z_{1} z_{1} z_{2}^{3}+8 \partial z_{1} z_{1} z_{2}^{2} z_{3}-4 \partial z_{1} z_{1} z_{2} z_{3}^{2}+\partial z_{1} z_{2}^{4} \\
& -2 \partial z_{1} z_{2}^{3} z_{3}+\partial z_{1} z_{2}^{2} z_{3}^{2}-\partial z_{2} z_{1}^{4}+2 \partial z_{2} z_{1}^{3} z_{2} \\
& -4 \partial z_{2} z_{1}^{2} z_{2}^{2}+4 \partial z_{2} z_{1}^{2} z_{2} z_{3}-\partial z_{2} z_{1}^{2} z_{3}^{2}+4 \partial z_{2} z_{1} z_{2}^{3} \\
& -6 \partial z_{2} z_{1} z_{2}^{2} z_{3}+2 \partial z_{2} z_{1} z_{2} z_{3}^{2}+\partial z_{3} z_{1}^{4}-2 \partial z_{3} z_{1}^{3} z_{2} \\
& +3 \partial z_{3} z_{1}^{2} z_{2}^{2}-2 \partial z_{3} z_{1}^{2} z_{2} z_{3}-2 \partial z_{3} z_{1} z_{2}^{3}+2 \partial z_{3} z_{1} z_{2}^{2} z_{3} \\
& +z_{1}^{4} z_{2}^{2}-2 z_{1}^{4} z_{2} z_{3}+z_{1}^{4} z_{3}^{2}-2 z_{1}^{3} z_{2}^{3} \\
& +4 z_{1}^{3} z_{2}^{2} z_{3}-2 z_{1}^{3} z_{2} z_{3}^{2}+z_{1}^{2} z_{2}^{4}-2 z_{1}^{2} z_{2}^{3} z_{3}+z_{1}^{2} z_{2}^{2} z_{3}^{2} .
\end{aligned}
$$

Regularizator:

$$
\begin{gathered}
\mathbf{v}^{1}=(1,0,0), \quad Q^{1}[\mathbf{y}]=2 y_{1}-y_{2}, \\
\mathbf{v}^{2}=(0,1,0), \quad Q^{2}[\mathbf{y}]=2 y_{2}-y_{1}-2 y_{3}, \\
\mathbf{v}^{3}=(0,0,1), \quad Q^{3}[\mathbf{y}]=y_{3}-y_{2} .
\end{gathered}
$$

4.7. Riccati operators of age $|2,4,6\rangle$. Second case.

Riccatians:

$$
R_{2}[\mathbf{z}]=\partial z_{1}+\partial z_{2}+2 \partial z_{3}+2 z_{1}^{2}-z_{1} z_{2}+z_{2}^{2}-2 z_{2} z_{3}+2 z_{3}^{2}
$$




$$
\begin{aligned}
& R_{4}[\mathbf{z}]=10 \partial^{3} z_{1}+6 \partial^{3} z_{2}+8 \partial^{3} z_{3}+20 \partial^{2} z_{1} z_{1}-6 \partial^{2} z_{1} z_{2} \\
& -10 \partial^{2} z_{2} z_{1}+12 \partial^{2} z_{2} z_{2}-8 \partial^{2} z_{2} z_{3}-12 \partial^{2} z_{3} z_{2}+16 \partial^{2} z_{3} z_{3} \\
& +19\left(\partial z_{1}\right)^{2}-18 \partial z_{1} \partial z_{2}-8 \partial z_{1} \partial z_{3}+11\left(\partial z_{2}\right)^{2}-24 \partial z_{2} \partial z_{3} \\
& +16\left(\partial z_{3}\right)^{2}-2 \partial z_{1} z_{1}^{2}+10 \partial z_{1} z_{1} z_{2}-6 \partial z_{1} z_{2}^{2}+8 \partial z_{1} z_{2} z_{3} \\
& -8 \partial z_{1} z_{3}^{2}+2 \partial z_{2} z_{1}^{2}-6 \partial z_{2} z_{1} z_{2}-2 \partial z_{2} z_{2}^{2}+12 \partial z_{2} z_{2} z_{3} \\
& -8 \partial z_{2} z_{3}^{2}-8 \partial z_{3} z_{1}^{2}+8 \partial z_{3} z_{1} z_{2}-8 \partial z_{3} z_{2} z_{3}-z_{1}^{4} \\
& +2 z_{1}^{3} z_{2}-3 z_{1}^{2} z_{2}^{2}+8 z_{1}^{2} z_{2} z_{3}-8 z_{1}^{2} z_{3}^{2}+2 z_{1} z_{2}^{3} \\
& -8 z_{1} z_{2}^{2} z_{3}+8 z_{1} z_{2} z_{3}^{2}-z_{2}^{4}+4 z_{2}^{3} z_{3}-4 z_{2}^{2} z_{3}^{2}, \\
& R_{6}[\mathbf{z}]=5 \partial^{5} z_{1}+\partial^{5} z_{2}+10 \partial^{4} z_{1} z_{1}-\partial^{4} z_{1} z_{2}-5 \partial^{4} z_{2} z_{1} \\
& +2 \partial^{4} z_{2} z_{2}-2 \partial^{4} z_{3} z_{2}+37 \partial^{3} z_{1} \partial z_{1}-11 \partial^{3} z_{1} \partial z_{2} \\
& -12 \partial^{3} z_{1} \partial z_{3}-17 \partial^{3} z_{2} \partial z_{1}+7 \partial^{3} z_{2} \partial z_{2}-4 \partial^{3} z_{2} \partial z_{3} \\
& -8 \partial^{3} z_{3} \partial z_{1}-8 \partial^{3} z_{3} \partial z_{2}+30\left(\partial^{2} z_{1}\right)^{2}-20 \partial^{2} z_{1} \partial^{2} z_{2} \\
& -20 \partial^{2} z_{1} \partial^{2} z_{3}+5\left(\partial^{2} z_{2}\right)^{2}-10 \partial^{2} z_{2} \partial^{2} z_{3}-3 \partial^{3} z_{1} z_{1}^{2} \\
& +11 \partial^{3} z_{1} z_{1} z_{2}-7 \partial^{3} z_{1} z_{2}^{2}+12 \partial^{3} z_{1} z_{2} z_{3}-12 \partial^{3} z_{1} z_{3}^{2} \\
& +3 \partial^{3} z_{2} z_{1}^{2}-7 \partial^{3} z_{2} z_{1} z_{2}-\partial^{3} z_{2} z_{2}^{2}+6 \partial^{3} z_{2} z_{2} z_{3} \\
& -4 \partial^{3} z_{2} z_{3}^{2}-8 \partial^{3} z_{3} z_{1}^{2}+8 \partial^{3} z_{3} z_{1} z_{2}-4 \partial^{3} z_{3} z_{2} z_{3} \\
& -6 \partial^{2} z_{1} \partial z_{1} z_{1}+27 \partial^{2} z_{1} \partial z_{1} z_{2}+18 \partial^{2} z_{1} \partial z_{2} z_{1}-27 \partial^{2} z_{1} \partial z_{2} z_{2} \\
& +20 \partial^{2} z_{1} \partial z_{2} z_{3}-24 \partial^{2} z_{1} \partial z_{3} z_{1}+24 \partial^{2} z_{1} \partial z_{3} z_{2}-40 \partial^{2} z_{1} \partial z_{3} z_{3} \\
& +23 \partial^{2} z_{2} \partial z_{1} z_{1}-24 \partial^{2} z_{2} \partial z_{1} z_{2}+8 \partial^{2} z_{2} \partial z_{1} z_{3}-19 \partial^{2} z_{2} \partial z_{2} z_{1} \\
& -6 \partial^{2} z_{2} \partial z_{2} z_{2}+18 \partial^{2} z_{2} \partial z_{2} z_{3}+12 \partial^{2} z_{2} \partial z_{3} z_{1}+12 \partial^{2} z_{2} \partial z_{3} z_{2} \\
& -20 \partial^{2} z_{2} \partial z_{3} z_{3}-40 \partial^{2} z_{3} \partial z_{1} z_{1}+24 \partial^{2} z_{3} \partial z_{1} z_{2}-16 \partial^{2} z_{3} \partial z_{1} z_{3} \\
& +20 \partial^{2} z_{3} \partial z_{2} z_{1}+6 \partial^{2} z_{3} \partial z_{2} z_{2}-16 \partial^{2} z_{3} \partial z_{2} z_{3}-12 \partial^{2} z_{3} \partial z_{3} z_{2} \\
& -6\left(\partial z_{1}\right)^{3}+22\left(\partial z_{1}\right)^{2} \partial z_{2}-20\left(\partial z_{1}\right)^{2} \partial z_{3}-22 \partial z_{1}\left(\partial z_{2}\right)^{2} \\
& +40 \partial z_{1} \partial z_{2} \partial z_{3}-16 \partial z_{1}\left(\partial z_{3}\right)^{2}-2\left(\partial z_{2}\right)^{3}+12\left(\partial z_{2}\right)^{2} \partial z_{3} \\
& -16 \partial z_{2}\left(\partial z_{3}\right)^{2}-6 \partial^{2} z_{1} z_{1}^{3}+9 \partial^{2} z_{1} z_{1}^{2} z_{2}-9 \partial^{2} z_{1} z_{1} z_{2}^{2} \\
& +24 \partial^{2} z_{1} z_{1} z_{2} z_{3}-24 \partial^{2} z_{1} z_{1} z_{3}^{2}+\partial^{2} z_{1} z_{2}^{3}-4 \partial^{2} z_{1} z_{2}^{2} z_{3} \\
& +4 \partial^{2} z_{1} z_{2} z_{3}^{2}+3 \partial^{2} z_{2} z_{1}^{3}-5 \partial^{2} z_{2} z_{1}^{2} z_{2}+8 \partial^{2} z_{2} z_{1}^{2} z_{3} \\
& +5 \partial^{2} z_{2} z_{1} z_{2}^{2}-20 \partial^{2} z_{2} z_{1} z_{2} z_{3}+12 \partial^{2} z_{2} z_{1} z_{3}^{2}-2 \partial^{2} z_{2} z_{2}^{3} \\
& +6 \partial^{2} z_{2} z_{2}^{2} z_{3}-4 \partial^{2} z_{2} z_{2} z_{3}^{2}+4 \partial^{2} z_{3} z_{1}^{2} z_{2}-16 \partial^{2} z_{3} z_{1}^{2} z_{3} \\
& -4 \partial^{2} z_{3} z_{1} z_{2}^{2}+16 \partial^{2} z_{3} z_{1} z_{2} z_{3}+2 \partial^{2} z_{3} z_{2}^{3}-4 \partial^{2} z_{3} z_{2}^{2} z_{3} \\
& -6\left(\partial z_{1}\right)^{2} z_{1}^{2}+6\left(\partial z_{1}\right)^{2} z_{1} z_{2}-5\left(\partial z_{1}\right)^{2} z_{2}^{2}+20\left(\partial z_{1}\right)^{2} z_{2} z_{3} \\
& -20\left(\partial z_{1}\right)^{2} z_{3}^{2}+2 \partial z_{1} \partial z_{2} z_{1}^{2}-14 \partial z_{1} \partial z_{2} z_{1} z_{2}+40 \partial z_{1} \partial z_{2} z_{1} z_{3} \\
& +10 \partial z_{1} \partial z_{2} z_{2}^{2}-40 \partial z_{1} \partial z_{2} z_{2} z_{3}+24 \partial z_{1} \partial z_{2} z_{3}^{2}+8 \partial z_{1} \partial z_{3} z_{1}^{2} \\
& +16 \partial z_{1} \partial z_{3} z_{1} z_{2}-80 \partial z_{1} \partial z_{3} z_{1} z_{3}-8 \partial z_{1} \partial z_{3} z_{2}^{2}+32 \partial z_{1} \partial z_{3} z_{2} z_{3} \\
& -4\left(\partial z_{2}\right)^{2} z_{1}^{2}+10\left(\partial z_{2}\right)^{2} z_{1} z_{2}-20\left(\partial z_{2}\right)^{2} z_{1} z_{3}-6\left(\partial z_{2}\right)^{2} z_{2}^{2}
\end{aligned}
$$




$$
\begin{gathered}
+12\left(\partial z_{2}\right)^{2} z_{2} z_{3}-4\left(\partial z_{2}\right)^{2} z_{3}^{2}+16 \partial z_{2} \partial z_{3} z_{1}^{2}-28 \partial z_{2} \partial z_{3} z_{1} z_{2} \\
+40 \partial z_{2} \partial z_{3} z_{1} z_{3}+12 \partial z_{2} \partial z_{3} z_{2}^{2}-16 \partial z_{2} \partial z_{3} z_{2} z_{3}-16\left(\partial z_{3}\right)^{2} z_{1}^{2} \\
+16\left(\partial z_{3}\right)^{2} z_{1} z_{2}-4\left(\partial z_{3}\right)^{2} z_{2}^{2}+2 \partial z_{1} z_{1}^{2} z_{2}^{2}-8 \partial z_{1} z_{1}^{2} z_{2} z_{3} \\
+8 \partial z_{1} z_{1}^{2} z_{3}^{2}-6 \partial z_{1} z_{1} z_{2}^{3}+24 \partial z_{1} z_{1} z_{2}^{2} z_{3}-24 \partial z_{1} z_{1} z_{2} z_{3}^{2} \\
+2 \partial z_{1} z_{2}^{4}-8 \partial z_{1} z_{2}^{3} z_{3}+8 \partial z_{1} z_{2}^{2} z_{3}^{2}-2 \partial z_{2} z_{1}^{4} \\
+4 \partial z_{2} z_{1}^{3} z_{2}-4 \partial z_{2} z_{1}^{2} z_{2}^{2}+4 \partial z_{2} z_{1}^{2} z_{2} z_{3}+4 \partial z_{2} z_{1} z_{2}^{3} \\
-12 \partial z_{2} z_{1} z_{2}^{2} z_{3}+8 \partial z_{2} z_{1} z_{2} z_{3}^{2}+4 \partial z_{3} z_{1}^{4}-8 \partial z_{3} z_{1}^{3} z_{2} \\
+8 \partial z_{3} z_{1}^{2} z_{2}^{2}-8 \partial z_{3} z_{1}^{2} z_{2} z_{3}-4 \partial z_{3} z_{1} z_{2}^{3}+8 \partial z_{3} z_{1} z_{2}^{2} z_{3} \\
\quad+z_{1}^{4} z_{2}^{2}-4 z_{1}^{4} z_{2} z_{3}+4 z_{1}^{4} z_{3}^{2}-2 z_{1}^{3} z_{2}^{3} \\
+8 z_{1}^{3} z_{2}^{2} z_{3}-8 z_{1}^{3} z_{2} z_{3}^{2}+z_{1}^{2} z_{2}^{4}-4 z_{1}^{2} z_{2}^{3} z_{3}+4 z_{1}^{2} z_{2}^{2} z_{3}^{2} .
\end{gathered}
$$

Regularizator:

$$
\begin{gathered}
\mathbf{v}^{1}=(1,0,0), \quad Q^{1}[\mathbf{y}]=y_{1}-\frac{1}{2} y_{2}, \\
\mathbf{v}^{2}=(0,1,0), \quad Q^{2}[\mathbf{y}]=y_{2}-\frac{1}{2} y_{1}-y_{3} \\
\mathbf{v}^{3}=(0,0,1), \quad Q^{3}[\mathbf{y}]=2 y_{3}-y_{2} .
\end{gathered}
$$

5. Riccatians and simple Lie algebras. Let us try to explane why the list of Riccatians given in the previous section is so interesting to us. First reason is associated with the fact that this list obtained as a solution of a typical analytic problem (in whose formulation has not been used any notion of symmetry), has purely Lie algebraic interpretation. This assertion (obtained after analyzing the results of previous section) we can formulate in the form of the following theorem.

THEOREM 5.1. There exists a correspondence between systems of simple Riccati operators of genus 1 and simple Lie algebras. Any simple Riccati operator of age $\left|n_{1}, \ldots, n_{r}\right\rangle$ with $r \leq 3$ and $n_{r} \leq 6$ is associated with some simple Lie algebra $\mathcal{L}_{r}$ of rank $r$. The corresponding systems of Riccatians have the following properties:

1. The product of degrees of particular Riccatians forming the system is equal to the dimension of the Weyl group for algebra $\mathcal{L}_{r}$.

2. The degrees of particular Riccatians coincide with degrees of independent Casimir invariants for algebra $\mathcal{L}_{r}$. In particular, each system of Riccatians contains Riccatian of second degree.

3. The regularizator of the system consists of $r$ pairs $\left\{\mathbf{v}^{n}, Q^{n}[\mathbf{y}]\right\}, \quad n=1, \ldots, r$ in which all vectors $\mathbf{v}^{n}$ are linearly independent.

4. It is possible to choose such a basis in r-dimensional vector space (in which function $\mathbf{z}$ takes its values), in which all the regularizing vectors $\mathbf{v}_{n}$ become orthonormal. In this basis (which we shall call canonical) the regularizing polynomials take the form

$$
Q^{n}[\mathbf{y}]=\sum_{m=1}^{r}\left(\pi^{n}, \pi^{m}\right) y_{m}
$$


where $\pi^{n}, n=1, \ldots, r$ are simple roots of algebra $\mathcal{L}_{r}$ and $(, \quad)$ denotes their scalar product.

5. In the canonical basis the second-order Riccatian has the form

$$
R_{2}[\mathbf{z}]=\sum_{i=1}^{r}\left(\pi^{i}, \pi^{i}\right) \partial z_{i}+\sum_{i, k=1}^{r}\left(\pi^{i}, \pi^{k}\right) z_{i} z_{k} .
$$

6. The leading term of any Riccatian (i.e. term not containing the derivatives) has the form $g^{i_{1}, \ldots, i_{n}} z_{i_{1}} \cdots z_{i_{n}}$ where $n$ is the degree of the Riccatian and $g^{i_{1}, \ldots, i_{n}}$ is an invariant tensor of the rank $r$ generating the Cartan part of nth order Casimir invariant for algebra $\mathcal{L}_{r}$.

This theorem is very interesting from purely marthematical point of view, because it reveals an intriguing relationship between the analytic proprties of Riccati operators and their hidden symmetry properties. Beyond any doubt, the fact that the structure of Casimir invariants of simple Lie algebras is encoded in the systems of Riccatians and can be obtained in absolutely non-algebraic way, has a great theoretical significance and deserves a careful study.

Conjecture 5.1. There exist one-to one correspondence between all systems of Riccatians of genus 1 and all simple Lie algebras. The restriction to Lie algebras of ranks $r \leq 3$ and to Riccati operators of ages $\left|n_{1}, \ldots, n_{r}\right\rangle$ with $r \leq 3$ and $n_{r} \leq 6$, used in theorem 5.1 , is not necessary.

In fact, we already have a proof of this conjecture for algebras $A_{r}, B_{r}, C_{r}$ of arbitrary rank $r$ and also for algebra $D_{4}$. First three series have been analyzed by methods differing from those used in the present paper, while the $D_{4}$ case has been studied in the spirit of section $3\left({ }^{5}\right)$.

Theorem 5.1 and its generalizations are not the only reason for which the nonequivalent systems of Riccatians might be interesting to us. The second and, in our opinion, the main reason follows from the following theorem.

TheOREM 5.2. Let $R_{n_{1}}\left[z_{1}(\lambda), \ldots, z_{r}(\lambda)\right], \ldots, R_{n_{r}}\left[z_{1}(\lambda), \ldots, z_{r}(\lambda)\right]$ be a system of Riccatians associated with a simple Lie algebra $\mathcal{L}_{r}$ and represented in a canonical form. Then the solution of Problem 2.1 has the following form:

$$
\begin{gathered}
z_{a}(\lambda)=F_{a}(\lambda)+\sum_{i=1}^{M_{a}} \frac{1}{\lambda-\xi_{i}^{a}}, \\
c_{n_{a}}(\lambda)=R_{n_{a}}\left[F_{1}(\lambda)+\sum_{i=1}^{M_{1}} \frac{1}{\lambda-\xi_{i}^{1}}, \ldots, F_{r}(\lambda)+\sum_{i=1}^{M_{r}} \frac{1}{\lambda-\xi_{i}^{r}}\right],
\end{gathered}
$$

where $M_{a}, a=1, \ldots, r$ are arbitrary non-negative integers, and the parameters $\xi_{i}^{a}, i=$ $1, \ldots, M_{a}, a=1, \ldots, r$ satisfy the system of equations

$$
F^{a}\left(\xi_{i}^{a}\right)+\sum_{b} \sum_{k=1}^{M^{b}} \frac{\left(\pi^{a}, \pi^{b}\right)}{\xi_{i}^{a}-\xi_{k}^{b}}=0, \quad i=1, \ldots, M_{a}, a=1, \ldots, r .
$$

$\left({ }^{5}\right)$ The paper with this proof is still in preparation. 
In particular, the eigenvalues $c_{2}(\lambda)$ are given by the formula

$$
\begin{gathered}
c_{2}(\lambda)=\sum_{a, b=1}^{r}\left(\pi^{a}, \pi^{b}\right)\left(F_{a}(\lambda)+\sum_{i=1}^{M_{a}} \frac{1}{\lambda-\xi_{i}^{a}}\right)\left(F_{b}(\lambda)+\sum_{i=1}^{M_{b}} \frac{1}{\lambda-\xi_{i}^{a}}\right) \\
+\sum_{a=1}^{r}\left(\pi^{a}, \pi^{a}\right)\left(F_{a}(\lambda)+\sum_{i=1}^{M_{a}} \frac{\hbar}{\lambda-\xi_{i}^{a}}\right)^{\prime} .
\end{gathered}
$$

For any set of numbers $M_{1}, \ldots, M_{r}$ the equations (5.4) have a finite set of solutions and therefore, the spectrum of the generalized Gaudin problem is infinite and discrete.

Proof. The proof of this theorem immediately follows from general theorem 2.1 and concrete forms of regularizing polynomials and second-order Riccatians given by formulas (5.1) and (5.2).

In next section we demonstrate that this solution exactly coincides with solution of the generalized Gaudin spectral problem associated with algebra $\mathcal{L}_{r}$.

6. Generalized Gaudin spectral problem. In this section we consider Gaudin models associated with arbitrary simple Lie algebras. Let $\mathcal{L}_{r}$ be a simple Lie algebra of rank $r$ and dimension $d$. The corresponding Gaudin algebra, which we denote by $\mathcal{G}\left[\mathcal{L}_{r}\right]$, is an infinite-dimensional extension of algebra $\mathcal{L}_{r}$. Its covariant generators we denote by $S^{A}(\lambda)$, where $\lambda$ is a complex parameter playing the role of a continuous index, and $A$ is a discrete index. The commutation relations for these operators can be written in the form

$$
\left[S^{A}(\lambda), S^{B}(\mu)\right]=-\hbar C^{A B}{ }_{C} \frac{S^{C}(\lambda)-S^{C}(\mu)}{\lambda-\mu},
$$

in which $C^{A B}{ }_{C}$ are structure constants of algebra $\mathcal{L}_{r}$.

The Cartan-Weyl decomposition of algebra $\mathcal{L}_{r}$ induces an analogous decomposition of the Gaudin algebra $\mathcal{G}[r]$. Correspondingly, the set of $d$ generators $S^{A}(\lambda)$ can be divided into three subsets consisting of raising and lowering operators, $S^{\alpha}(\lambda), \alpha \in R_{ \pm r}$, associated with positive and negative roots of algebra $\mathcal{L}_{r}$ and $r$ neutral operators $S^{a}(\lambda), a \in N_{r}$.

The lowest weight representations of algebra $\mathcal{G}\left[\mathcal{L}_{r}\right]$ are determined by the formulas

$$
\begin{gathered}
S^{a}(\lambda)|0\rangle=F^{a}(\lambda)|0\rangle, \quad a \in N_{r}, \\
S^{\alpha}(\lambda)|0\rangle=0, \quad \alpha \in R_{-r},
\end{gathered}
$$

where $|0\rangle$ is the lowest weight vector and $F^{a}(\lambda)$ are covariant components of the lowest weight $\vec{F}(\lambda)$. The representation space is then defined as

$$
W_{\vec{F}(\lambda)}=\text { linear span of vectors }\left\{S^{\alpha_{1}}\left(\lambda_{1}\right) \cdots S^{\alpha_{n}}\left(\lambda_{n}\right)|0\rangle\right\}
$$

with arbitrary $\alpha_{1}, \ldots, \alpha_{n} \in R_{+r}$ and $\lambda_{1}, \ldots, \lambda_{n}$ for each $n=0,1,2, \ldots$.

Consider the operator

$$
C_{2}(\lambda)=g_{A B} S^{A}(\lambda) S^{B}(\lambda)
$$

which belongs to the universal enveloping algebra of algebra $\mathcal{G}\left[\mathcal{L}_{r}\right]$ and has the form similar to the form of the second-order Casimir operator for algebra $\mathcal{L}_{r}$. As before, we 
call it the Casimir-Gaudin operator. Not being a Casimir invariant for algebra $\mathcal{G}\left[\mathcal{L}_{r}\right]$, the operator $C_{2}(\lambda)$ forms however a commutative family

$$
\left[C_{2}(\lambda), C_{2}(\mu)\right]=0 .
$$

This property suggests to interpret $C_{2}(\lambda)$ as a generating function of commuting integrals of motion for some quantum system. It can be shown that, in contrast with the $s l(2)$ case, the functions $C_{2}(\lambda)$ do not contain enough number of commuting integrals of motion sufficient for claiming that the corresponding quantum system is completely integrable. The question of a complete integrability of these quantum problems is still open except the problems associated with algebra $s l(3)$ (see discussion in Section 7). Fortunately, irrespective of a concrete answer to this question, one can demonstrate that the spectral problem for them admits a simple and elegant solution. Taking the representation space of Gaudin algebra $\mathcal{G}\left[\mathcal{L}_{r}\right]$ as an analog of the space of states, we can formulate the following analog of the Schródinger problem for operators $C_{2}(\lambda)$ :

Problem 6.1. Find all solutions of the spectral equation

$$
C_{2}(\lambda) \phi=c_{2}(\lambda) \phi, \quad \phi \in W_{\vec{F}(\lambda)}
$$

provided that the lowest weight $F(\lambda)=F^{1}(\lambda), \ldots, F^{r}(\lambda)$ is given.

Definition 6.1. The equation (6.6) is called the $\mathcal{L}_{r}$ Gaudin spectral equation and the models described by "hamiltonians" $C_{2}(\lambda)$ we refer to as $\mathcal{L}_{r}$ Gaudin models.

THEOREM 6.1. The solution of problem 6.1 for algebras $A_{r}, B_{r}, C_{r}, D_{r}, E_{6}$ and $E_{7}$ has the following form

$$
\begin{gathered}
\phi=\prod_{a=1}^{r} \prod_{i=1}^{M_{a}} S^{\pi^{a}}\left(\xi_{i}^{a}\right)|0\rangle+\ldots \\
c_{2}(\lambda)=\sum_{a, b=1}^{r}\left(\pi^{a}, \pi^{b}\right)\left(F_{a}(\lambda)+\sum_{i=1}^{M_{a}} \frac{1}{\lambda-\xi_{i}^{a}}\right)\left(F_{b}(\lambda)+\sum_{i=1}^{M_{b}} \frac{1}{\lambda-\xi_{i}^{a}}\right) \\
+\sum_{a=1}^{r}\left(\pi^{a}, \pi^{a}\right)\left(F_{a}(\lambda)+\sum_{i=1}^{M_{a}} \frac{1}{\lambda-\xi_{i}^{a}}\right)^{\prime} .
\end{gathered}
$$

where $\xi_{i}^{a}$ are the numbers satisfying the system of equations

$$
\sum_{b=1}^{r} \sum_{k=1, k \neq i}^{M_{b}} \frac{\left(\pi^{a}, \pi^{b}\right)}{\xi_{i}^{a}-\xi_{k}^{b}}=F^{a}\left(\xi_{i}^{a}\right),
$$

where $a=1, \ldots, r$ and $i=1, \ldots, M_{a}$. For any set of numbers $M_{1}, \ldots, M_{r}$ the equations (6.8) have a finite set of solutions and therefore, the spectrum of the generalized Gaudin problem is infinite and discrete.

The proof of this theorem can be found in refs. [Ushveridze 1990, 1992, 1994]. For the proof for only classical Lie algebras $A_{r}, B_{r}, C_{r}, D_{r}$ see also [Jurčo 1989]. As far as we know, the solutions of the Gaudin spectral problems associated with three exceptional Lie algebras $G_{2}, F_{4}, E_{8}$ are not yet found. 
7. Conclusion. Comparing the results of sections 5 and 6 we can make sure that the spectrum of the Riccatian $R_{2}[\mathbf{z}(\lambda)]$ associated with algebra $\mathcal{L}_{r}$ exactly coincides with the spectrum of the second-order Casimir - Gaudin operator $C_{2}(\lambda)$ constructed from generators of Gaudin algebra $\mathcal{G}\left[\mathcal{L}_{r}\right]$. Moreover, the Riccatian $R_{2}[\mathbf{z}(\lambda)]$ itself has the same form as the Cartan part of th normally-ordered $\left({ }^{6}\right)$ operator $C_{2}(\lambda)$. We can go further and show that the higher Riccatians $R_{n_{i}}[\mathbf{z}(\lambda)]$ also coincide with Cartan parts of normallyordered higher-order Casimir - Gaudin operators $C_{n_{i}}(\lambda)$ (i.e. operators constructed from generators of Gaudin algebra $\mathcal{G}\left[\mathcal{L}_{r}\right]$ and having the same structure as independent Casimir invariants of algebra $\mathcal{L}_{r}$.)

Evidently, all these coincidences cannot be accidental and should manifest of a deep relationship between Riccati and Gaudin spectral problems.

Two things immediately come in ones head. First is that the generalized Gaudin models are integrable and the operators $C_{n_{i}}(\lambda), i=1, \ldots, r$ (or their slight deformations) form a complete set of quantum integrals of motion $\left({ }^{7}\right)$ whose spectra coincide with spectra of Riccatians. Second thing is that the Gaudin models admit separation of variables and the resulting multi-parameter spectral equations appearing after the separation are somehow related to generalized Riccati equations $\left({ }^{8}\right)$.

It turns out, however, that the verification of these assumptions is far from being a simple matter. The main difficulty lies in the fact that the operators $C_{n_{1}}(\lambda), \ldots, C_{n_{r}}(\lambda)$ do not generally form commutative families $\left({ }^{9}\right)$, and thus, cannot be considered as generating functions for quantum integrals of motion $\left({ }^{10}\right)$. There are two possible explanations of this situation: 1) the Gaudin models are non-integrable on the quantum level, and 2) they are integrable but the form of additional integrals of motion is more complicated $\left({ }^{11}\right)$.

In our opinion, the second possibility seems more realistic, and in order to prove this, we can start with the systems of Riccati equations and try to obtain the Gaudin models by means of the inverse method of separation of variables (exactly in the same way as for the $A_{1}$ case). This program will be realized in next publication. Here we only anonce some preliminary results which, in our opinion, are rather interesting.

- The systems of generalized Riccati equations can be separated, i.e. transformed into systems of $r$ different equations, each of which contains only one of functions $z_{1}(\lambda), \ldots$ $\ldots, z_{r}(\lambda)$.

- The separated systems can be linearized by means of the substitution $z_{i}(\lambda)=$ $\psi_{i}^{\prime}(\lambda) / \psi_{i}(\lambda)$, after which one obtains a system of linear multi-parameter spectral equations.

$\left({ }^{6}\right)$ Normal ordering means that all lowering operators are transfered to the right.

$\left({ }^{7}\right)$ As in the classical case.

$\left.{ }^{8}\right)$ As in the $s l(2)$ case.

$\left({ }^{9}\right)$ Except the cases of algebras $A_{1}$ and $A_{2}$.

$\left({ }^{10}\right)$ For example, this can be easily demonstrated by direct calculation of commutators for algebra $A_{3}$.

$\left.{ }^{11}\right)$ The method for constructing additional integrals of motion for quantum models associated with sl(n) solutions of Yang - Baxter equation, given in [Kulish and Sklyanin 1982], is wrong because it is based on the use of non-invertible R-matrices. 
- The main feature of obtained equations is that they depend on spectral parameters non-linearly (except the cases of algebras $A_{1}$ and $A_{2}$ ).

- As a consequence of this non-linearity, the completely integrable models obtained from these multi-parameter spectral equations after applying to them the inverse method of separation of variables are non-linear in the sense that, along with ordinary (linear) integrals of motion, they contain a set of nonlinear ones, represented by non-linear operators in Hilbert space! Evidently, such models cannot be considered as ordinary quantum models.

- The integrals of motion for these models, which we denote by $\hat{C}_{n_{i}}(\lambda)$, have the same spectra as the Riccatians $R_{n_{i}}[\mathbf{z}(\lambda)]$.

Unfortunately, up to now it is not clear whether these "pseudo-quantal" completely integrable models do actually have some relation to Gaudin models. In order to prove this we should try to demonstrate that the linear operator $\hat{C}_{2}(\lambda)$ constructed in such a way is nothing else than the second-order Casimir - Gaudin operator $C_{2}(\lambda)$. If it will be done then one can claim that the remaining integrals of motion of Gaudin models are some non-linear deformations of high-order Casimir - Gaudin operators! We hope to return to this interesting question in next publication.

In conclusion I would like to thank my colleagues from the Theoretical Department for many valuable comments and remarks.

\section{References}

[1] M. Gaudin, J. Physique 37 (1976), 1087-98.

[2] —, La Fonction d'Onde de Bethe (Paris: Masson), 1983.

[3] V. E. Jurčo, 1989 J. Math. Phys. 30 (1989), 1289-91.

[4] G. A. Korn and T. M. Korn, Mathematical Handbook (New York: McGraw-Hill), 1971.

[5] P. P. Kulish and E. K. Sklyanin, Lect. Not. Phys. 151 (1982), 61-119.

[6] E. K. Sklyanin, Zap. Nauchn. Semin. LOMI 164 (1987), 151-170 (in Russian).

[7] - , Preprint of Helsinki University HU-TFT-91-51, Helsinki (see also hep-th/9211111), 1991.

[8] —, Preprint of Cambridge University NI-92013, Cambridge, 1992.

[9] A. G. Ushveridze, Sov. J. Part. Nucl. 20 (1989), 1185-245.

[10] - , Preprint of Georgian Institute of Physics FTT-16, Tbilisi, 1990 (in Russian).

[11] -, Sov. J. Part. Nucl. 23 (1992), 25-51.

[12] —, Quasi-exactly solvable models in quantum mechanics (Bristol: IOP Publishing), 1994. 